\title{
PERRON'S SOLUTIONS FOR TWO-PHASE FREE BOUNDARY PROBLEMS WITH DISTRIBUTED SOURCES
}

\author{
DANIELA DE SILVA, FAUSTO FERRARI, AND SANDRO SALSA
}

\begin{abstract}
We use Perron method to construct a weak solution to a two-phase free boundary problem with right-hand-side. We thus extend the results in [C3] for the homogeneous case.
\end{abstract}

Dedicated to Enzo Mitidieri on his 60-th birthday

\section{INTRODUCTION}

Let $\Omega$ be a bounded Lipschitz domain in $\mathbb{R}^{n}$ and let $A=A(x)$ be a symmetric matrix with Hölder continuous coefficients in $\Omega$, which is uniformly elliptic, i.e.

$$
\lambda|\xi|^{2} \leq \sum_{i, j=1}^{n} a_{i j}(x) \xi_{i} \xi_{j} \leq \Lambda|\xi|^{2}, \quad \forall x \in \Omega, \quad \xi \in \mathbb{R}^{n}
$$

for some $0<\lambda \leq \Lambda$. Denote by

$$
\mathcal{L}:=\operatorname{div}(A(x) \nabla \cdot) .
$$

Let $f_{1}, f_{2} \in L^{\infty}(\Omega)$. We consider the following two-phase inhomogeneous free boundary problem (f.b.p. in the sequel)

$$
\begin{cases}\mathcal{L} u=f_{1}, & \text { in } \Omega^{+}(u)=\{u>0\} \\ \mathcal{L} u=f_{2} \chi_{\{u<0\}} & \text { in } \Omega^{-}(u)=\{u \leq 0\}^{\circ} \\ u_{\nu}^{+}=G\left(u_{\nu}^{-}, x, \nu\right) & \text { on } F(u)=\partial\{u>0\} \cap \Omega .\end{cases}
$$

Here $\nu=\nu(x)$ denotes the unit normal to $F(u)$ at $x$, pointing towards $\Omega^{+}(u)$. The function $G(\beta, x, \nu)$ is strictly increasing in $\beta$, Lipschitz continuous in the first and in the third argument, Hölder continuous in the second argument, and moreover $G(0):=\inf _{x \in \Omega,|\nu|=1} G(0, x, \nu)>0$. Conormal derivatives $\left\langle A \nabla u^{ \pm}, \nu\right\rangle$ can be equally considered instead of normal derivatives.

Problems of this kind arise in several contexts, see [DFSs1] for a list.

In this paper, our main purpose is to construct a weak solution assuming given boundary data, via Perron method, extending the results of the seminal paper [C3] in the homogeneous case. A different approach to prove existence for a specific class of free boundary problems in divergence form can be found for example in [TE].

Before stating our main result, we give the definition of weak solution of problem (1.1).

Given a continuous function $v$ on $\Omega$, we say that a point $x_{0} \in F(v)$ is regular from the right (resp. left) if there is a ball $B \subset \Omega^{+}(v)$ (resp. $B \subset \Omega^{-}(v)$ ), such

D.D. is partially supported by NSF grant DMS-1301535. F. F. is supported by the ERC starting grant project 2011 EPSILON (Elliptic PDEs and Symmetry of Interfaces and Layers for Odd Nonlinearities) 277749 and by RFO grant, Università di Bologna, Italy. 
that $\bar{B} \cap F(v)=\left\{x_{0}\right\}$. In what follows, $\nu=\nu\left(x_{0}\right)$ represents the unit normal to $\partial B$ at $x_{0}$ pointing toward $\Omega^{+}(v)$.

Definition 1.1. We say that $u \in C(\Omega)$ is a weak solution of the f.b.p. (1.1) if:

a) $\mathcal{L} u=f_{1}$ in $\Omega^{+}(u)$ and $\mathcal{L} u=f_{2} \chi_{\{u<0\}}$ in $\Omega^{-}(u)$, in the usual weak sense;

b) $u$ satisfies the free boundary condition in (1.1) in the following sense:

(i) If $x_{0} \in F(u)$ is regular from the right with touching ball $B$ then

$$
u^{+}(x) \geq \alpha\left\langle x-x_{0}, \nu\right\rangle^{+}+o\left(\left|x-x_{0}\right|\right) \quad \text { in } B, \text { with } \alpha \geq 0
$$

and

$$
u^{-}(x) \leq \beta\left\langle x-x_{0}, \nu\right\rangle^{-}+o\left(\left|x-x_{0}\right|\right) \quad \text { in } B^{c}, \text { with } \beta \geq 0
$$

with equality along every non-tangential domain, and

$$
\alpha \leq G\left(\beta, x_{0}, \nu\left(x_{0}\right)\right) .
$$

(ii) If $x_{0} \in F(u)$ is regular from the left with touching ball $B$, then

$$
\begin{gathered}
u^{-}(x) \geq \beta\left\langle x-x_{0}, \nu\right\rangle^{-}+o\left(\left|x-x_{0}\right|\right) \quad \text { in } B, \text { with } \beta \geq 0 \\
u^{+}(x) \leq \alpha\left\langle x-x_{0}, \nu\right\rangle^{+}+o\left(\left|x-x_{0}\right|\right) \quad \text { in } B^{c}, \text { with } \alpha \geq 0
\end{gathered}
$$

with equality along every non-tangential domain, and

$$
\alpha \geq G\left(\beta, x_{0}, \nu\left(x_{0}\right)\right) .
$$

Note that (i) (resp. (ii)) expresses a supersolution (resp. subsolution) condition at points regular from the right (resp. left). While this definition slightly differs from the one in [C3], it is indeed equivalent to it (see ([CS]).

Our solution is constructed as the infimum over an admissible class of supersolutions $\mathcal{F}$.

Definition 1.2. A function $w \in \mathcal{F}$ if $w \in C(\bar{\Omega})$ and if

(a) $w$ is a weak solution to

$$
\mathcal{L} w \leq f_{1} \quad \text { in } \Omega^{+}(w) \quad \text { and } \quad \mathcal{L} w \leq f_{2} \chi_{\{w<0\}} \quad \text { in } \Omega^{-}(w) .
$$

(b) If $x_{0} \in F(u)$ is regular from the left, then near $x_{0}$,

$$
\begin{aligned}
& w^{+} \leq \alpha\left\langle x-x_{0}, \nu\left(x_{0}\right)\right\rangle^{+}+o\left(\left|x-x_{0}\right|\right), \quad \alpha \geq 0, \\
& w^{-} \geq \beta\left\langle x-x_{0}, \nu\left(x_{0}\right)\right\rangle^{-}+o\left(\left|x-x_{0}\right|\right), \quad \beta \geq 0,
\end{aligned}
$$

with

$$
\alpha<G\left(\beta, x_{0}, \nu\left(x_{0}\right)\right) \text {. }
$$

(c) If $x_{0} \in F(w)$ is not regular from the left, then near $x_{0}$,

$$
w(x)=o\left(\left|x-x_{0}\right|\right) .
$$

We also need to introduce a minorant subsolution. We say that a locally Lipschitz function $\underline{u}$, defined in $\Omega$, is a minorant if:

a) $\underline{u}$ is a weak solution to

$$
\mathcal{L} \underline{u} \geq f_{1} \quad \text { in } \Omega^{+}(\underline{u}) \quad \text { and } \quad \mathcal{L} \underline{u} \geq f_{2} \chi_{\{\underline{u}<0\}} \text { in } \Omega^{-}(\underline{u}) .
$$

b) Every $x_{0} \in F(\underline{u})$ is regular from the right and near $x_{0}$,

$$
\begin{aligned}
& \underline{u}^{-} \leq \beta\left\langle x-x_{0}, \nu\left(x_{0}\right)\right\rangle^{-}+o\left(\left|x-x_{0}\right|\right), \\
& \underline{u}^{+} \geq \alpha\left\langle x-x_{0}, \nu\left(x_{0}\right)\right\rangle^{+}+o\left(\left|x-x_{0}\right|\right),
\end{aligned}
$$


with

$$
\alpha>G\left(\beta, x_{0}, \nu\left(x_{0}\right)\right) .
$$

We are now ready to state our main result.

Theorem 1.3. Let $\phi$ be a continuous function on $\partial \Omega$ and $\underline{u}$ be a minorant of our free boundary problem, with boundary data $\phi$. Then

$$
u=\inf \{w: w \in \mathcal{F}, w \geq \underline{u} \text { in } \bar{\Omega}\}
$$

is a solution to (1.1) such that $u=\phi$ on $\partial \Omega$, as long as the set on the right is non-empty.

Concerning the regularity of the free boundary, we prove the following standard result.

Theorem 1.4. The free boundary $F(u)$ has finite $(n-1)$-dimensional Hausdorff measure. More precisely, there exists a universal constant $r_{0}>0$ such that for every $r<r_{0}$, for every $x_{0} \in F(u)$,

$$
\mathcal{H}^{n-1}\left(F(u) \cap B_{r}\left(x_{0}\right)\right) \leq r^{n-1} .
$$

Moreover, denoting with $F^{*}(u)$ the reduced free boundary

$$
\mathcal{H}^{n-1}\left(F(u) \backslash F^{*}(u)\right)=0 .
$$

In a forthcoming paper we shall address further regularity properties of the free boundary. In particular, compactness properties of the minimal solutions constructed in Theorem 1.3 and the flatness result in [DFS4] will imply the following corollary, new even in the homogeneous case.

Theorem 1.5. $F(u)$ is a $C^{1, \gamma}$ surface in a neighborhood of $H^{n-1}$ a.e. point $x_{0} \in$ $F(u)$.

The paper follows the main guidelines of [C3], although the presence of a distributed source requires to face new situations and requires new delicate arguments, especially in Sections 4 and 5. The organization is as follows. In Section 2 we prove some preliminary lemmas frequently used throughout the paper. In Section 3 we prove that $u^{+}$is Lipschitz continuous. Then in Section 4 we show that $u$ is Lipschitz continuous and it satisfies the equation in both $\Omega^{+}(u)$ and $\Omega^{-}(u)$. Linear growth near the free boundary and the non-degeneracy of $u^{+}$are proved in Section 5 . The following section, Section 6 , is devoted to the proof that $u$ satisfies the free boundary condition in the supersolution sense (part b(i). in Definition 1.1). Finally in Section 7 we prove that $u$ satisfies the free boundary condition in the subsolution sense (part b(ii). in Definition 1.1) and hence it is a weak solution to our problem. We conclude our paper with the regularity result in Theorem 1.4 in Section 8.

Throughout the paper, constants depending possibly only on $[A]_{C^{0, \gamma},},\left\|f_{1}\right\|_{\infty},\left\|f_{2}\right\|_{\infty}$, $\lambda, \Lambda, G(0), n$ are called universal. Whenever a constant depends on other parameters, that dependance will be explicitly noted. Finally, for standard regularity theory for weak solution to divergence form equations, we refer the reader to [GT].

\section{Preliminaries}

In this section we introduce some notation and prove some useful lemmas which will be used several times in the paper. 
Notation. As usual, $B_{r}\left(x_{0}\right)$ denotes the ball in $\mathbb{R}^{n}$ of radius $r$ and center $x_{0}$. When $x_{0}=0$ we omit the dependence on $x_{0}$. Also, throughout the paper we will use the following notation for rescalings of size $r$ around $x_{0}$ :

$$
\begin{aligned}
& u_{r}(x):=\frac{u\left(x_{0}+r x\right)}{r}, \quad x \in \Omega_{r}:=\frac{\left\{x-x_{0}: x \in \Omega\right\}}{r}, \\
& A_{r}(x)=A\left(x_{0}+r x\right), \quad f^{r}(x)=f\left(x_{0}+r x\right), \quad \mathcal{L}_{r}=\operatorname{div}\left(A_{r}(x) \nabla \cdot\right) \\
& G_{r}(\alpha, x, \nu)=G\left(\alpha, x_{0}+r x, \nu\left(x_{0}+r x\right)\right) .
\end{aligned}
$$

Finally, as in the Introduction we denote

$$
G(0):=\inf _{x \in \Omega,|\nu|=1} G(0, x, \nu)>0 .
$$

Lemma 2.1. Let $v \in C\left(\overline{B_{r}\left(x_{0}\right)}\right), v \geq 0$ for $r \leq 1$. Assume that

$$
\mathcal{L} v=f \in L^{\infty} \quad \text { in } B_{r}\left(x_{0}\right)
$$

and

$$
v\left(y_{0}\right)=0, \quad y_{0} \in \partial B_{r}\left(x_{0}\right) .
$$

Denote by $\nu$ the inner unit normal to $\partial B_{r}\left(x_{0}\right)$ at $y_{0}$. Then,

$$
v(x) \geq \alpha\left\langle x-y_{0}, \nu\right\rangle^{+}+o\left(\left|x-y_{0}\right|\right)
$$

with

$$
\alpha \geq \bar{c} \frac{v\left(x_{0}\right)}{r}-\bar{C} r\|f\|_{\infty}
$$

and $\bar{c}, \bar{C}>0$ depending on $[A]_{0, \gamma}, \lambda, \Lambda, n$.

Proof. Let

$$
v_{r}(x)=\frac{v\left(x_{0}+r x\right)}{r}, \quad x \in B_{1}
$$

Then,

$$
v_{r} \geq 0 \quad \text { in } B_{1}, \quad v_{r}\left(y_{r}\right)=0, \quad y_{r} \in \partial B_{1}
$$

and

$$
\mathcal{L}_{r} v_{r}=r f^{r} \quad \text { in } B_{1} .
$$

Notice that $A_{r}(x)=A\left(x_{0}+r x\right)$ has the same ellipticity constants as $A$ and its $C^{0, \gamma}$ norm is controlled by the $C^{0, \gamma}$ norm of $A$.

Call $\|f\|_{\infty}=M$. By Harnack inequality,

$$
\inf _{B_{1 / 2}} v_{r} \geq c\left(v_{r}(0)-r M\right)
$$

Now, denote with $\xi, \eta$ the solutions to the the following problems:

$$
\begin{gathered}
\mathcal{L}_{r} \xi=-1 \quad \text { in } B_{1} \backslash \overline{B_{1 / 2}} \\
\xi=0 \quad \text { on } \partial B_{1 / 2}, \quad \xi=0 \quad \text { on } \partial B_{1}, \\
\mathcal{L}_{r} \eta=0 \quad \text { in } B_{1} \backslash \overline{B_{1 / 2}} \\
\eta=1 \quad \text { on } \partial B_{1 / 2}, \quad \eta=0 \quad \text { on } \partial B_{1} .
\end{gathered}
$$

Call

$$
c_{1}=\left.\xi_{\nu}\right|_{\partial B_{1}}>0 \quad c_{2}=\left.\eta_{\nu}\right|_{\partial B_{1}}>0,
$$

with $\nu$ the inner unit normal to $\partial B_{1}$. Notice that $c_{1}$ depends only on $[A]_{C^{0, \gamma}}, \lambda, \Lambda, n$.

Define,

$$
\phi:=c\left(v_{r}(0)-r M\right) \eta+r M \xi \quad \text { in } B_{1} \backslash \overline{B_{1 / 2}} \text {. }
$$


Then,

$$
\mathcal{L}_{r} \phi=-r M \geq r f^{r} \quad \text { in } B_{1} \backslash \overline{B_{1 / 2}},
$$

and

$$
\phi \leq v_{r} \quad \text { on } \partial B_{1} \cup \partial B_{1 / 2} \text {. }
$$

Thus,

$$
\phi \leq v_{r} \quad \text { in } B_{1} \backslash \overline{B_{1 / 2}}
$$

and hence

$$
v_{r}(x) \geq\left(c\left(v_{r}(0)-r M\right) c_{1}+r M c_{2}\right)\left\langle x-y_{r}, \nu\right\rangle^{+}+o\left(\left|x-y_{r}\right|\right),
$$

which gives the desired result.

Next we prove the following asymptotic developments lemmas.

Lemma 2.2. Let $\Omega$ be an open set, $0 \in \partial \Omega$. Assume that $B_{\rho}\left(-\rho e^{1}\right) \subset R^{n} \backslash \bar{\Omega}$. Let $u$ be a nonnegative Lipschitz function in $B_{1} \cap \bar{\Omega}$, satisfying $\mathcal{L} u=f$ in $B_{1} \cap \Omega$ and $u=0$ on $\partial \Omega \cap B_{1}$.

Then there exists $\alpha \geq 0$ such that

$$
u(x)=\alpha x_{1}+o(|x|) \quad \text { as } x \rightarrow 0, x \in \bar{\Omega} \cap B_{1} .
$$

In particular, if $\alpha>0$, then along $\partial \Omega$,

$$
x_{1}=o(|x|) \quad \text { as } x \rightarrow 0, x \in \partial \Omega \cap B_{1}
$$

that is $\partial \Omega$ is tangent to the hyperplane $x_{1}=0$.

Proof. We may assume that $\rho<1 / 3$. We change variables by setting

$$
y=T(x)=\frac{e^{1}}{\rho}-\frac{x+\rho e^{1}}{\left|x+\rho e^{1}\right|^{2}}
$$

and define $v(y)=u\left(T^{-1}(y)\right)$. Then $T(0)=0$, and the exterior of the ball $B_{\rho}\left(-\rho e^{1}\right)$ is mapped onto $B_{1 / \rho}\left(e^{1} / \rho\right) \backslash\left\{e^{1} / \rho\right\}$. Thus $\Omega^{\prime}=T(\Omega) \subset B_{1 / \rho}\left(e^{1} / \rho\right)$ and $\Omega^{\prime} \cap B_{2} \subset B_{2}^{+}=\left\{y \in B_{2}: y_{1}>0\right\}$.

Note also that,

$$
y_{1}=\left(\frac{2}{\rho^{2}}-1\right) x_{1}+o(|x|) .
$$

Moreover, $v$ is Lipschitz in $\bar{\Omega}^{\prime} \cap B_{2}, v=0$ on $\partial \Omega^{\prime} \cap B_{2}$ and

$$
\mathcal{L}^{\prime} v=\operatorname{div}\left(A^{\prime}(y) \nabla v\right)=f^{\prime}(y) \equiv f\left(T^{-1}(y)\right) \cdot|\operatorname{det} J| \quad \text { in } \Omega^{\prime} \cap B_{2}
$$

where $A^{\prime}=J A J^{\top} \cdot|\operatorname{det} J|, J$ being the Jacobian of $T^{-1}$. Note that if $A$ is symmetric then $A^{\prime}$ is symmetric and

$$
c(\rho, \lambda) I \leq A^{\prime}(y) \leq C(\rho, \Lambda) I \quad \text { in } \bar{\Omega}^{\prime} \cap B_{2} .
$$

Extend $v$ by zero in $B_{1}$ outside $\Omega^{\prime}$. Then (still calling $v$ the extended function), $\mathcal{L}^{\prime} v \geq-\left\|f^{\prime}\right\|_{\infty}$ in $B_{2}^{+}$(in a weak sense). We also have $v(y) \leq C y_{1}$ in $B_{3 / 2}^{+}$(compare with the solution of $\mathcal{L}^{\prime} z=-\left\|f^{\prime}\right\|_{\infty}, z=v$ on $\left.\partial B_{2}^{+}\right)$.

Now, let $w=w(x)$ be the $\mathcal{L}^{\prime}$-harmonic measure in $B_{2}^{+}$of $S_{2}^{+}=\partial B_{2} \cap\left\{y_{1}>0\right\}$. Then, by Hopf principle and standard regularity theory,

$$
y_{1} c_{1} \leq w(y) \leq c_{2} y_{1} \quad \text { in } \bar{B}_{1}^{+}
$$


with $c_{1}, c_{2}$ positive and universal, and, for some universal $\gamma>0$,

$$
w(y)=\gamma y_{1}+o(|y|) \text { as } y \rightarrow 0, y \in B_{1}^{+} .
$$

Let now for $k \geq 1$, integer,

$$
m_{k}=\inf \left\{m: v(y) \leq m w(y) \quad \text { for every } y \in \bar{\Omega}^{\prime} \cap B_{1 / k}\right\} .
$$

Then $\left\{m_{k}\right\}$ is non increasing and $m_{k} \rightarrow m_{\infty} \geq 0$. Moreover

$$
v(y) \leq m_{\infty} w(y)+o(|y|) \quad \text { as } y \rightarrow 0, y \in \bar{\Omega}^{\prime} \cap B_{1} .
$$

We claim that equality holds in (2.5). If not, there exist $\delta>0$ and a sequence $\left\{y_{j}\right\} \in \Omega^{\prime} \cap B_{1}$ such that $r_{j}=\left|y_{j}\right| \rightarrow 0$ and

$$
v\left(y_{j}\right) \leq m_{\infty} w\left(y_{j}\right)-\delta r_{j} .
$$

Since both $v$ and $w$ are Lipschitz, we can write

$$
W(y) \equiv m_{\infty} w(y)-v(y) \geq \delta r_{j} / 2 \quad \text { on } B_{c r_{j}}\left(y_{j}\right) \cap S_{r_{j}}^{+}
$$

with $c$ depending on $m_{\infty}$ and the Lipschitz constants of $v$ and $w$.

On the other hand, (2.5) implies that

$$
W(y) \geq-\sigma_{j} r_{j} \quad \text { on } S_{r_{j}}^{+}
$$

with $\sigma_{j} \rightarrow 0$. Rescale by setting

$$
W_{j}(y)=m_{\infty} \frac{w\left(r_{j} y\right)}{r_{j}}-\frac{v\left(r_{j} y\right)}{r_{j}}=\frac{W\left(r_{j} y\right)}{r_{j}} \quad y \in B_{1}^{+} .
$$

Note that (2.3) still holds for $w\left(r_{j} y\right) / r_{j}$. Then $W_{j}(y)=0$ on $y_{1}=0, W_{j}(y) \geq-\sigma_{j}$ on $S_{1}^{+}, W_{j}(y) \geq \delta / 2$ on $B_{c}\left(y_{j} / r_{j}\right) \cap S_{1}^{+}$. Moreover, setting $\mathcal{L}_{j}^{\prime}=\operatorname{div}\left(A^{\prime}\left(r_{j} y\right) \nabla\right)$,

$$
\mathcal{L}_{j}^{\prime} W_{j} \leq r_{j}\left\|f^{\prime}\right\|_{\infty} \quad \text { in } B_{1}^{+} .
$$

By Hopf principle and standard comparison, in $B_{1 / 2}^{+}$we can write

$$
W_{j}(y) \geq\left(-c_{3} \sigma_{j}-c_{4} r_{j}\left\|f^{\prime}\right\|_{\infty}+c_{5} \delta / 2\right) y_{1}
$$

with $c_{3}, c_{5}$ universal and $c_{5}$ depending on the Lipschitz constant of $v$. For $j$ large enough, we get, say

$$
m_{\infty} w_{j}(y)-v_{j}(y) \geq \frac{\delta}{100} y_{1} .
$$

Rescaling back and using (2.3), we get a contradiction to the definition of $m_{\infty}$. Thus we have equality in (2.5) and taking into account (2.4), we get

$$
v(x)=\gamma m_{\infty} y_{1}+o(|y|) \quad \text { as } x \rightarrow 0, x \in \bar{\Omega}^{\prime} \cap B_{1} .
$$

Going back to the original variables, from (2.2), we get

$$
u(x)=\alpha x_{1}+o(|x|) \text { as } x \rightarrow 0, x \in \bar{\Omega} \cap B_{1}
$$

with $\alpha=\left(\frac{2}{\rho^{2}}-1\right) \gamma m_{\infty}$.

Lemma 2.3. Let $\Omega$ be an open set, $0 \in \partial \Omega$. Assume that

$$
B_{\rho}\left(\rho e^{1}\right) \subset \Omega
$$

Let $u$ be a nonnegative Lipschitz function in $B_{2} \cap \bar{\Omega}$, satisfying $\mathcal{L} u=f$ in $B_{2} \cap \Omega$ and $u=0$ on $\partial \Omega \cap B_{2}$. 
Then there exists $\alpha \geq 0$ such that

$$
u(x)=\alpha x_{1}+o(|x|) \quad \text { as } \quad x \rightarrow 0, x \in B_{\rho}\left(\rho e^{1}\right) .
$$

Proof. After a smooth change of variables (e.g. flattening the surface ball) which leaves both the origin and the normal direction at 0 fixed, we may replace (2.8) by

$$
B_{2}^{+} \subset \Omega
$$

always with $0 \in \partial \Omega$. We keep the same notation $u$ and $\mathcal{L}$ for the transformed $u$ and the new operator, which is uniformly elliptic with ellipticity constant of the same order of $\lambda, \Lambda$. As in Lemma 2.2, let $w=w(x)$ be the $\mathcal{L}$-harmonic measure in $B_{2}^{+}$ of $S_{2}^{+}=\partial B_{2} \cap\left\{y_{1}>0\right\}$. For $k \geq 1$, integer, define

$$
\alpha_{k}=\sup \left\{\beta: u(x) \geq \beta w(x) \quad \text { for every } x \in B_{1 / k}^{+}\right\} .
$$

Then $\left\{\alpha_{k}\right\}$ is nondecreasing and $\alpha_{k} \rightarrow \alpha \geq 0$. Moreover

$$
u(x) \geq \alpha w(x)+o(|x|) \quad \text { as } x \rightarrow 0, x \in B_{1}^{+} .
$$

We claim that equality holds in (2.5). If not, there exist $\delta>0$ and a sequence $\left\{x_{j}\right\} \in B_{1}^{+}$such that $r_{j}=\left|x_{j}\right| \rightarrow 0$ and

$$
u\left(x_{j}\right)-\alpha w\left(x_{j}\right) \geq \delta r_{j} .
$$

By Lipschitz continuity, we can write

$$
U(x) \equiv u(x)-\alpha w(x) \geq \delta r_{j} / 2 \quad \text { on } B_{c r_{j}}\left(x_{j}\right) \cap S_{r_{j}}^{+}
$$

with $c$ depending on $\alpha$ and the Lipschitz constants of $u$ and $w$.

On the other hand, (2.5) implies that

$$
U(x) \geq-\sigma_{j} r_{j} \quad \text { on } S_{r_{j}}^{+}
$$

with $\sigma_{j} \rightarrow 0$. Rescale by setting

$$
U_{j}(x)=\frac{u\left(r_{j} x\right)}{r_{j}}-\alpha \frac{w\left(r_{j} x\right)}{r_{j}}=\frac{U\left(r_{j} x\right)}{r_{j}} \quad x \in B_{1}^{+} .
$$

Then $U_{j}(0)=0, U_{j}(x) \geq-\sigma_{j}$ on $S_{1}^{+}, U_{j}(x) \geq \delta / 2$ on $B_{c}\left(x_{j} / r_{j}\right) \cap S_{1}^{+}$. Moreover, setting $\mathcal{L}_{j}=\operatorname{div}\left(A\left(r_{j} x\right) \nabla\right)$,

$$
\mathcal{L}_{j} U_{j} \leq r_{j}\|f\|_{\infty} \quad \text { in } B_{1}^{+} .
$$

By Hopf principle and standard arguments, in $B_{1 / 2}^{+}$we can write, for $j$ large

$$
U_{j}(x) \geq\left(-c \sigma_{j}-c_{0} r_{j}\|f\|_{\infty}+C \delta / 2\right) x_{1} \geq \frac{\delta}{100} x_{1} .
$$

Rescaling back and using (2.3), we get a contradiction to the definition of $\alpha$.

Remark 2.4. We remark that the expansions in the lemmas above remain valid if we replace the assumption that $u$ is Lipschitz with the existence of a touching ball at 0 both from the right and from the left. 


\section{LiPSCHITZ REGULARITY OF $u^{+}$.}

In this section we prove that $u^{+}$is Lipschitz continuos. In order to follow the strategy developed in [C3], we need the following "almost-monotonicity" formula, see $[\mathrm{MP}]$. For other types of related almost-monotonicity formulae, see also [TZ].

Proposition 3.1. Let $u_{i}, i=1,2$ be continuous functions in the unit ball $B_{1}$ that satisfy

$$
u_{i} \geq 0, \quad \mathcal{L} u_{i} \geq-1, \quad u_{1} \cdot u_{2}=0 \quad \text { in } \quad B_{1} .
$$

Then there exist universal constants $C_{0}$ and $r_{0}$, such that the functional

$$
\Phi(r):=r^{-4} \int_{B_{r}} \frac{\left|\nabla u_{1}\right|^{2}}{|x|^{n-2}} \int_{B_{r}} \frac{\left|\nabla u_{2}\right|^{2}}{|x|^{n-2}} d x
$$

satisfies

$$
\Phi(r) \leq C_{0}\left(1+\left\|u_{1}\right\|_{L^{2}\left(B_{1}\right)}^{2}+\left\|u_{2}\right\|_{L^{2}\left(B_{1}\right)}^{2}\right)^{2}
$$

for $0<r<r_{0}$.

Remark 3.2. We remark that, by Fubini's theorem

$$
\int_{B_{R}} \frac{\left|\nabla u_{i}\right|^{2}}{|x|^{n-2}} d x=R^{2-n} \int_{B_{R}}\left|\nabla u_{i}\right|^{2} d x+(n-2) R^{-2} \int_{0}^{r}\left(\int_{B_{r}}\left|\nabla u_{i}\right|^{2}\right) r^{1-n} d r .
$$

Remark 3.3. We remark that if $v$ satisfies $\mathcal{L} v \geq-M$ say in $B_{1}^{+}(v)$, then $\mathcal{L} v^{+} \geq-M$ in $B$. This follows by standard arguments. Indeed, if $\psi_{\varepsilon}(t)$ is a convex increasing function such that $\psi_{\varepsilon}(t)=0$ for $t \leq \varepsilon$, then it is easy to see that

$$
\mathcal{L} \psi_{\varepsilon}(v) \geq \psi^{\prime}\left(u_{\varepsilon}\right) \mathcal{L} v \geq-M \quad \text { in } B_{1} .
$$

The desired result follows by approximating $t^{+}$with a sequence of $\psi_{\varepsilon}$.

The next lemma is the first step towards proving that $u^{+}$is Lipschitz. The standard technique of harmonic replacement cannot be applied in our case, as we are not imposing any sign condition on the right-hand-side $f_{1}$. We bypass this difficulty solving an obstacle-type problem.

Lemma 3.4. Let $w \in \mathcal{F}$, then there exists $\tilde{w} \in \mathcal{F}$ such that

(i) $\mathcal{L} \tilde{w}=f_{1}$ in $\Omega^{+}(\tilde{w})$

(ii) $\tilde{w}^{+} \leq w, \tilde{w}^{-}=w$

(iii) $\tilde{w} \geq \underline{u}, \quad \tilde{w}=\phi$ on $\partial \Omega$.

Proof. Let $w \in \mathcal{F}$. For notational simplicity call $\Omega^{+}=\Omega^{+}(w)$ and set

$$
\mathcal{S}=\left\{v \in C\left(\bar{\Omega}^{+}\right): \mathcal{L} v \geq f_{1} \chi_{\{v>0\}} \text { in } \Omega^{+}, v \geq 0 \text { in } \Omega^{+}, v=w \text { on } \partial \Omega^{+}\right\} .
$$

Notice that $\mathcal{S} \neq \emptyset$ since $\underline{u}^{+} \in \mathcal{S}$. Also, if $v \in \mathcal{S}$ then $v \leq w$ in $\Omega^{+}$. Define,

$$
\tilde{w}:=\sup \mathcal{S} \text {. }
$$

Then $\tilde{w} \leq w$ and solves the obstacle problem (see $[\mathrm{KS}]$ )

$$
\left\{\begin{array}{l}
\mathcal{L} \tilde{w}=f_{1} \quad \text { in }\{\tilde{w}>0\}, \quad \tilde{w} \geq 0 \quad \text { in } \Omega^{+} \\
\tilde{w}=w \quad \text { on } \partial \Omega^{+} .
\end{array}\right.
$$

By the regularity theory for the obstacle problem we conclude that $\tilde{w}$ is locally $C^{1, \gamma}$ in $\Omega^{+}$(see $\left.[\mathrm{T}]\right)$.

Extend $\tilde{w}$ to $\bar{\Omega}$ by setting

$$
\tilde{w}=w \quad \text { in } \bar{\Omega} \cap\{w \leq 0\} .
$$


Hence by definition, $\tilde{w} \geq \underline{u}$ on $\Omega$ and $\tilde{w}=g$ on $\partial \Omega$.

To conclude that $\tilde{w} \in \mathcal{F}$ we only need to show that $\tilde{w}$ satisfies the free boundary condition in the sense of Definition 1.2.

Let $x_{0} \in F(\tilde{w})$, then either $x_{0} \in F(w)$ or $x_{0} \in \Omega^{+} \cap \partial\{\tilde{w}=0\}$. In the latter case, by the $C^{1, \gamma}$ regularity of $\tilde{w}$ we immediately obtain that the free boundary condition in satisfied, possibly with $\alpha=\beta=0$ (recall $G(0)>0$.) If $x_{0} \in F(w)$ then the conclusion follows immediately from the fact that $\tilde{w} \leq w$ in $\Omega^{+}$and $\tilde{w}=w$ otherwise.

The following result is a consequence of the weak monotonicity formula.

Theorem 3.5. Let $w \in \mathcal{F}$ and $\mathcal{L} w=f_{1}$ in $\Omega^{+}(w)$. Then, $w^{+}$is locally Lipschitz in $\Omega$. Moreover, denoting by

$$
G^{-1}(\alpha)=\inf _{x, \nu} G^{-1}(\alpha, x, \nu),
$$

for any $D \subset \subset \Omega, w^{+}$is Lipschitz in $D$ with Lipschitz constant $L_{D}$ satisfying

$$
L_{D} G^{-1}\left(L_{D}\right) \leq C\left(1+\left\|w^{+}\right\|_{L^{2}(D)}^{2}+\left\|w^{-}\right\|_{L^{2}(D)}^{2}\right)
$$

and $C$ depending on $D$.

Proof. Let $x_{0} \in F(w)$ be a regular point from the left where $w$ has the asymptotic development

$$
\begin{gathered}
w^{+}=\alpha\left\langle x-x_{0}, \nu\right\rangle^{+}+o\left(\left|x-x_{0}\right|\right), \quad \alpha>0 \\
w^{-} \geq \beta\left\langle x-x_{0}, \nu\right\rangle^{-}+o\left(\left|x-x_{0}\right|\right), \quad \beta \geq 0,
\end{gathered}
$$

with

Let us show that

$$
\alpha<G\left(\beta, x_{0}, \nu\right)
$$

$$
\alpha G^{-1}(\alpha) \leq C\left(1+\left\|w^{+}\right\|_{L^{2}}^{2}+\left\|w^{-}\right\|_{L^{2}}^{2}\right)^{2},
$$

with $C$ depending on $\operatorname{dist}\left(x_{0}, \partial \Omega\right)$. We will use Proposition 3.1. Notice that in view of Remark 3.3, the conclusion of Proposition 3.1 holds for $u_{1}=w^{+}, u_{2}=w^{-}$.

If $G^{-1}(\alpha)=0$, then there is nothing to prove. Thus, let $G^{-1}(\alpha)>0$ and let us prove that

$$
\alpha^{2} \beta^{2} \leq C\left(1+\left\|w^{+}\right\|_{L^{2}}^{2}+\left\|w^{-}\right\|_{L^{2}}^{2}\right)^{2}
$$

from which the desired inequality will follow.

For convenience, use coordinates $x=\left(x^{\prime}, y\right) \in \mathbb{R}^{n-1} \times \mathbb{R}$ and assume that $x_{0}=$ $(0,0), \nu=(0,1)$. Following [C3] pg. 587, one can estimate that as $s \rightarrow 0$,

$$
\int_{B_{s}}\left|\nabla w^{+}\right|^{2} d x \geq \int_{B_{s} \cap\{y>0\}}\left(\alpha^{2}-o(1)\right) d x
$$

and

$$
\int_{B_{s}}\left|\nabla w^{-}\right|^{2} d x \geq \int_{B_{s} \cap\{y>0\}}\left(\beta^{2}-o(1)\right) d x .
$$

Thus, by Remark 3.2, for all sufficiently small $s$, if $\Phi$ is the functional defined in Proposition 3.1

$$
\Phi(s) \geq c_{n} s^{-4} \int_{0}^{s}\left(\alpha^{2}-o(1)\right) r d r \int_{0}^{s}\left(\beta^{2}-o(1)\right) r d r
$$

with $c_{n}=16 / \omega_{n}^{2}\left(\omega_{n}\right.$ the measure of the unit sphere.) 
Hence, for all $r$ small $\alpha^{2} \beta^{2} \leq C(\Phi(r)+1)$ which together with the conclusion of Proposition 3.1 gives the desired estimate.

Now, let $x_{0} \in \Omega^{+}(w) \cap D$ and let

$$
\operatorname{dist}\left(x_{0}, F(w)\right)=\left|x_{0}-y_{0}\right|=r<\frac{1}{2} \operatorname{dist}(D, \partial \Omega)
$$

say $r \leq 1$.

To prove the result it is sufficient to prove the existence of a positive constant $M$ such that

$$
\frac{w\left(x_{0}\right)}{r} \leq M
$$

Suppose

$$
\frac{w\left(x_{0}\right)}{r}>M
$$

with $M$ to be specified later. By Lemma 2.1, we have that

$$
w(x) \geq \alpha_{M}\left\langle x-y_{0}, \nu\right\rangle^{+}+o\left(\left|x-y_{0}\right|\right)
$$

with

$$
\alpha_{M}=\bar{c} M-\bar{C} r\left\|f_{1}\right\|_{\infty} .
$$

For $M$ large $\alpha_{M}>0$ and $y_{0}$ is regular from the left. Then according to Lemma 2.4

$$
w(x)=\alpha\left\langle x-y_{0}, \nu\right\rangle^{+}+o\left(\left|x-y_{0}\right|\right)
$$

and $\alpha \geq \alpha_{M}$.

Hence we can apply the previous estimate and conclude that

$$
\alpha_{M} G^{-1}\left(\alpha_{M}\right) \leq C\left(1+\left\|w^{+}\right\|_{L^{2}}^{2}+\left\|w^{-}\right\|_{L^{2}}^{2}\right)^{2} .
$$

The contradiction follows for $M$ large, since $\alpha_{M} G^{-1}\left(\alpha_{M}\right) \rightarrow \infty$ as $M \rightarrow \infty$.

As a corollary of the two results above we obtain the following.

Corollary 3.6. $u^{+}$is locally Lipschitz and it satisfies

$$
\mathcal{L} u=f_{1} \quad \text { in } \Omega^{+}(u) .
$$

\section{The FUnCtion $u$ IS Lipschitz}

In this section we show that $u^{-}$is Lipschitz. First, we prove the following standard lemma.

Lemma 4.1. If $w_{1}, w_{2} \in \mathcal{F}$, then

$$
w^{*}=\min \left\{w_{1}, w_{2}\right\} .
$$

Proof. The fact that $\mathcal{L} w^{*}=f_{1}$ in $\Omega^{+}\left(w^{*}\right)$ and $\mathcal{L} w^{*}=f_{2} \chi_{\left\{w^{*}<0\right\}}$ follows from standard arguments. To prove that $w^{*}$ solves the free boundary condition in the sense of Definition 1.2 it is suffices to notice that $\Omega^{+}\left(w^{*}\right)=\Omega^{+}\left(w_{1}\right) \cap \Omega^{+}\left(w_{2}\right)$. Hence, any ball touching $F\left(w_{1}\right)$ or $F\left(w_{2}\right)$ from the left will also touch $F\left(w^{*}\right)$ from the left. Thus, if $x_{0}$ is not regular for $F\left(w^{*}\right)$, it cannot be regular for $F\left(w_{1}\right)$ and $F\left(w_{2}\right)$ either and near $x_{0}, w^{*}(x)=w_{1}(x)=w_{2}(x)=o\left(\left|x-x_{0}\right|\right)$. If $x_{0}$ is regular, the the asymptotic developments for $w^{*}$ come from those of $w_{1}$ or $w_{2}$ (possibly with $\alpha=\beta=0$ in case $x_{0}$ is not regular for either $F\left(w_{1}\right)$ or $F\left(w_{2}\right)$.) 
The proof of the Lipschitz continuity of $u^{-}$is based on the following replacement technique. The harmonic replacement technique in [C3] does not work in this context. Instead, we need to perform a replacement with solutions to obstacle-type problems.

Precisely, let $w \in \mathcal{F}$ and let $w\left(x_{0}\right)<0$. Let $B:=B_{R}\left(x_{0}\right)$ be a ball around $x_{0}$. Denote by

Define

$$
\Omega_{1}:=\Omega^{+}(w) \backslash \bar{B}
$$

$\mathcal{S}_{1}=\left\{v: \mathcal{L} v \geq f_{1} \chi_{\{v>0\}}\right.$ in $\Omega_{1}, v \geq 0$ in $\Omega_{1}, v=w$ on $\partial \Omega_{1} \backslash \partial B, v=0$ on $\left.\partial B\right\}$.

Notice that since $\underline{u}$ is locally Lipschitz and $\underline{u}\left(x_{0}\right) \leq w\left(x_{0}\right)<0, \underline{u}$ is strictly negative in $B$ for $R$ small. Hence $\underline{u}^{+} \in \mathcal{S}_{1}$ and $\mathcal{S}_{1}$ is non empty. Let

$$
w_{1}=\sup \mathcal{S}_{1} \text {. }
$$

Then $w_{1}$ solves the obstacle problem (see $[\mathrm{KS}, \mathrm{T}]$ )

$$
\mathcal{L} w_{1}=f_{1} \quad \text { in }\left\{w_{1}>0\right\}, \quad w_{1} \geq 0 .
$$

Analogously, define

$$
\mathcal{S}_{2}=\left\{v: \mathcal{L} v \geq-f_{2} \chi_{\{v>0\}} \text { in } B, v \geq 0 \text { in } B, v=w^{-} \text {on } \partial B\right\} .
$$

Clearly $\mathcal{S}_{2} \neq \emptyset$ since $w^{-} \in \mathcal{S}_{2}$. Let,

$$
w_{2}=\sup S_{2}
$$

Again, $w_{2}$ solves the obstacle problem

$$
\mathcal{L} w_{2}=-f_{2} \quad \text { in }\left\{w_{2}>0\right\}, \quad w_{2} \geq 0 .
$$

We define the "double-replacement" $\tilde{w}$ of $w$ in $B$ as follows

$$
\tilde{w}=\left\{\begin{array}{l}
w_{1} \quad \text { in } \bar{\Omega}_{1} \\
-w_{2} \quad \text { in } \bar{B} \\
w \quad \text { otherwise. }
\end{array}\right.
$$

By construction $\tilde{w} \leq w$. Indeed in $\Omega_{1}$ this follows by the maximum principle, while in $B$ it follows from the fact that $w^{-} \in \mathcal{S}_{2}$.

We wish to prove the following lemma.

Lemma 4.2. Let $w \in \mathcal{F}, w\left(x_{0}\right)=-h$. Then there exists $\epsilon$ (depending on $\operatorname{dist}\left(x_{0}, \partial \Omega\right)$ and $\underline{u}$ ) such that

(i) The double replacement $\tilde{w}$ of $w$ in $B_{\epsilon h}\left(x_{0}\right)$ belongs to $\mathcal{F}$ and $\underline{u} \leq \tilde{w} \leq w$.

(ii) $\mathcal{L} \tilde{w}=f_{2}$ and $\tilde{w}<0$ in $B_{\varepsilon h}\left(x_{0}\right)$ and

$$
|\nabla \tilde{w}| \leq \frac{C}{\epsilon}+\epsilon C\left\|f_{2}\right\|_{\infty} \quad \text { in } B_{\epsilon h / 2}\left(x_{0}\right) .
$$

Proof. We already noticed that $\tilde{w} \leq w$. Now we observe that $\tilde{w} \geq \underline{u}$. As already remarked, since $\underline{u}$ is locally Lipschitz and $\underline{u}\left(x_{0}\right)<-h, \underline{u}$ is strictly negative in $B:=B_{\epsilon h}\left(x_{0}\right)$ for $\epsilon$ small. Thus, $\underline{u}^{+} \in \mathcal{S}_{1}$ and $w_{1} \geq \underline{u}$. Also, by the maximum principle in $\left\{w_{2}>0\right\}$, it follows that $-w_{2} \geq \underline{u}$ in $B$. Hence $\tilde{w} \geq \underline{u}$. We denote with $-m=\min _{\bar{\Omega}} \underline{u}, m>0$. Notice that $h \leq m$.

Let us also observe that, for $\epsilon$ small, $w_{2}>0$ in $B_{\epsilon h}\left(x_{0}\right)$. Indeed if $\partial\left\{w_{2}>\right.$ $0\} \cap B_{\epsilon h}\left(x_{0}\right) \neq \emptyset$, then by the growth of the solution to the obstacle problem, we get

$$
w_{2}\left(x_{0}\right) \leq C(\varepsilon h)^{2}
$$


For $\epsilon$ small, this contradicts that $-w_{2}\left(x_{0}\right) \leq w\left(x_{0}\right)=-h$. In particular, it follows from (4.2) that

$$
\mathcal{L} \tilde{w}=f_{2} \quad \text { in } B_{\varepsilon h}\left(x_{0}\right) .
$$

Now, the fact that $\tilde{w}$ satisfies (a) in Definition 1.2 follows from (4.1)-(4.3) and standard arguments.

We need to verify that the free boundary condition is satisfied in the sense of part (b) in Definition 1.2. Let $\bar{x} \in F(\tilde{w})$. Then three possibilities can occur. If $x_{1} \in F(w)$, we use that $\tilde{w} \leq w$ and hence $\tilde{w}$ has the correct asymptotic behavior whether $x_{1}$ is regular or not (recall $G(0, \cdot, \cdot)>0$ ). If $x_{1} \in \partial\left\{w_{1}>0\right\} \cap \Omega^{+}(w)$ then by the regularity of the solution to the obstacle problem we get again that $\tilde{w}$ has the correct asymptotic behavior. Finally, we consider the case when $x_{1} \in \partial B \cap \Omega^{+}(w)$. Since $w^{+}$is locally Lipschitz, say with constant $L$ in $B_{d_{0} / 2}\left(x_{0}\right)$ we get that

$$
\tilde{w} \leq w^{+} \leq L \varepsilon h \text { in } B_{2 \varepsilon h}\left(x_{0}\right) .
$$

Let us rescale and using the notation in (2.1) call

$$
\tilde{w}_{\epsilon}(x)=\frac{\tilde{w}\left(x_{0}+\varepsilon h x\right)}{\varepsilon h} .
$$

Then,

$$
\tilde{w}_{\varepsilon} \leq L \text { in } B_{2} .
$$

Let us call $v_{1}, v_{2}$ the solutions to the the following problems:

$$
\begin{aligned}
\mathcal{L}_{\varepsilon} v_{1}=0 & \text { in } B_{2} \backslash \overline{B_{1}} \\
v_{1}=0 \quad \text { on } \partial B_{1}, & v_{1}=1 \quad \text { on } \partial B_{2}, \\
\mathcal{L}_{\varepsilon} v_{2}=-1 & \text { in } B_{2} \backslash \overline{B_{1}} \\
v_{2}=0 \quad \text { on } \partial B_{1}, & v_{2}=0 \quad \text { on } \partial B_{2} .
\end{aligned}
$$

Define,

$$
v:=L v_{1}+\varepsilon h M v_{2}
$$

with $M=\left\|f_{1}\right\|_{\infty}$. Then, applying the maximum principle in $\left(B_{2} \backslash \bar{B}_{1}\right) \cap \Omega^{+}\left(\tilde{w}_{\varepsilon}\right)$ we obtain that

$$
\tilde{w}_{\varepsilon}^{+} \leq v \quad \text { in } B_{2} \backslash \bar{B}_{1} .
$$

Thus,

$$
\tilde{w}_{\varepsilon}^{+} \leq \alpha\left\langle x-\left(x_{1}\right)_{\varepsilon}, \nu\left(\left(x_{1}\right)_{\varepsilon}\right)\right\rangle^{+}+o\left(\left|x-\left(x_{1}\right)_{\varepsilon}\right|\right)
$$

with

$$
\begin{aligned}
\alpha=\left(L c_{1}+\varepsilon h M c_{2}\right), \quad c_{1} & =\left.\left(v_{1}\right)_{\nu}\right|_{\partial B_{1}}, \quad c_{2}=\left.\left(v_{2}\right)_{\nu}\right|_{\partial B_{1}} \\
\left(x_{1}\right)_{\varepsilon} & =\frac{x_{1}-x_{0}}{\varepsilon h}
\end{aligned}
$$

and $\nu(y)$ the normal to $\partial B_{1}$ at $y$ pointing outside $B_{1}$.

In terms of $\tilde{w}$ this gives,

$$
\tilde{w}^{+} \leq \alpha\left\langle x-x_{1}, \nu\right\rangle^{+}+o\left(\left|x-x_{1}\right|\right), \quad \alpha \leq \bar{L},
$$

and $\nu$ the exterior unit normal to $\partial B_{\varepsilon h}\left(x_{0}\right)$ at $x_{1}$.

On the other hand, by Lemma 2.1 applied to $-\left(w_{2}\right)_{\varepsilon}$ we have that

$$
\tilde{w}_{\varepsilon}^{-}=-\left(w_{2}\right)_{\varepsilon} \geq \beta\left\langle x-\left(x_{1}\right)_{\varepsilon}, \nu\left(\left(x_{1}\right)_{\varepsilon}\right)\right\rangle^{-}+o\left(\left|x-\left(x_{1}\right)_{\varepsilon}\right|\right),
$$

with $\left(\bar{M}=\left\|f_{2}\right\|_{\infty}\right)$

$$
\beta \geq \frac{\bar{c}}{\varepsilon}-\bar{C} \varepsilon h \bar{M} .
$$


In terms of $\tilde{w}$ and for $\varepsilon$ small, this implies that

$$
\tilde{w}^{-} \geq \beta\left\langle x-x_{1}, \nu\right\rangle^{-}+o\left(\left|x-x_{1}\right|\right), \quad \beta \geq \frac{\bar{c}}{2 \varepsilon} .
$$

In view of (4.4)-(4.5), the free boundary condition is satisfied if we choose $\varepsilon$ small enough so that

$$
\bar{L}<\inf f_{x, \nu} G\left(\frac{\bar{c}}{\varepsilon}, \cdot, \cdot\right) .
$$

Finally, the estimate in (ii) follows from standard Schauder estimates and Harnack inequality.

We obtain the following immediate corollary.

Corollary 4.3. Let $x_{0}$ be a point where $u\left(x_{0}\right)=-h<0$. Then, there exists a nonincreasing sequence $\left\{\tilde{w}_{j}\right\} \subset \mathcal{F}, \tilde{w} \geq \underline{u}$, and $\varepsilon>0$, depending on $d_{0}=\operatorname{dist}\left(x_{0}, \partial \Omega\right)$, such that the following hold:

(i) $\tilde{w}_{k}\left(x_{0}\right) \searrow u\left(x_{0}\right)$;

(ii) $\mathcal{L} \tilde{w}_{k}=f_{2}$ and $\tilde{w}_{k}<0$ in $B_{\varepsilon h}\left(x_{0}\right)$;

(iii) For each $k, \tilde{w}_{k}$ is Lipschitz in $B_{\varepsilon h / 2}\left(x_{0}\right)$ with Lipschitz constant $L_{0}$ depending on $d_{0}$.

Finally, we can finish the proof that $u$ satisfies part a) in Definition 1.1.

Corollary 4.4. $u$ is locally Lipschitz in $\Omega$, continuous in $\bar{\Omega}, u=g$ on $\partial \Omega$. Moreover $u$ solves

$$
\mathcal{L} u=f_{2} \chi_{\{u<0\}}, \quad \text { in } \Omega^{-}(u) .
$$

Proof. Let $u\left(x_{0}\right)=-h<0$ and let $\left\{\tilde{w}_{k}\right\}$ be as in the lemma above. We want to prove that $\tilde{w}_{k} \searrow u$ uniformly, say on $B_{h \epsilon / 4}$. Indeed suppose by contradiction that there exists $x_{1} \in B_{\epsilon h / 4}\left(x_{0}\right)$ where $\tilde{w}\left(x_{1}\right)=\lim _{j \rightarrow \infty} \tilde{w}_{j}\left(x_{1}\right)>u\left(x_{1}\right)$. Then consider a new sequence $\left\{v_{j}\right\}_{j \in \mathbb{N}}$ converging to $u$ at $x_{1}$, and define $\left\{\tilde{u}_{k}\right\}$ as a replacement of $\left\{\min \left\{\tilde{v}_{k}, \tilde{w}_{k}\right\}\right\}_{k \in \mathbb{N}}$ in $B_{\epsilon h / 2}\left(x_{0}\right)$. Then $\lim _{k \rightarrow \infty} \tilde{u}_{k}=\tilde{u}$ decreasing with $\tilde{u} \leq \tilde{w}$ in $B_{\epsilon h / 2}\left(x_{0}\right), \tilde{u}\left(x_{0}\right)=\tilde{w}\left(x_{0}\right)$ and $\tilde{u}\left(x_{1}\right)<\tilde{w}\left(x_{1}\right)$. Moreover in $B_{\epsilon h / 4}\left(x_{0}\right)$

$$
\mathcal{L}(\tilde{w}-\tilde{u})=0,
$$

$\tilde{w}-\tilde{u} \geq 0$ and $(\tilde{w}-\tilde{u})\left(x_{0}\right)=0$ and by maximum principle it follows that $\tilde{w}-\tilde{u} \equiv 0$ obtaining a contradiction with $(\tilde{w}-\tilde{u})\left(x_{1}\right)>0$. As a consequence $u$ satisfies

$$
\mathcal{L} u=f_{2} \quad \text { in }\{u<0\} .
$$

Corollary 4.5. If $K$ is compactly contained in $\Omega$, then $u$ is uniform limit of a sequence of functions $\left\{w_{k}\right\}_{k \in \mathbb{N}} \subset \mathcal{F}$ in $K$. If $K \Subset \Omega^{-}(u),\left\{w_{k}\right\}_{k \in \mathbb{N}}$, may be taken non-positive in $\bar{K}$.

Proof. The first part follows from the fact that $\left\{w^{+}: w \in \mathcal{F}\right\}$ is equilipschitz in $\bar{K}$ and from the previous replacement technique.

By compactness, it is enough to prove the second part for balls $B_{\varepsilon}\left(x_{0}\right) \Subset \Omega^{-}(u)$, with $\varepsilon$ small enough. Let $w_{k} \searrow u$ uniformly in $\bar{B}_{2 \varepsilon}\left(x_{0}\right) \Subset \Omega^{-}(u)$. Let us rescale by $\varepsilon$ and use the notation in (2.1). Let $\eta, \xi$ solve the following problems:

$$
\begin{gathered}
\mathcal{L}_{\varepsilon} \eta=0 \quad \text { in } B_{2} \backslash \overline{B_{1}} \\
\eta=0 \quad \text { on } \partial B_{1}, \quad \eta=1 \quad \text { on } \partial B_{2},
\end{gathered}
$$




$$
\begin{gathered}
\mathcal{L}_{\varepsilon} \xi=-1 \quad \text { in } B_{2} \backslash \overline{B_{1}} \\
\xi=0 \quad \text { on } \partial B_{1}, \quad \xi=0 \quad \text { on } \partial B_{2} .
\end{gathered}
$$

Call

$$
c_{0}:=\left.\eta_{\nu}\right|_{\partial B_{1}}>0, \quad c_{1}:=\left.\xi_{\nu}\right|_{\partial B_{1}}>0
$$

with $\nu$ the unit normal to $\partial B_{1}$ pointing inward and let $c_{2}>0$ be such that

$$
c_{0} c_{2}<\frac{G(0)}{2}
$$

Define, $\left(M=\left\|f_{2}\right\|_{\infty}\right.$, say $\left.M>0\right)$

$$
v:=\varepsilon M \xi+c_{2} \eta \quad \text { in } B_{2} \backslash \overline{B_{1}}, \quad v \equiv 0 \quad \text { on } B_{1} .
$$

Then,

$$
\mathcal{L}_{\varepsilon} v=-\varepsilon M \leq \varepsilon f_{2}^{\varepsilon} \quad \text { in } B_{2} \backslash \overline{B_{1}} .
$$

Since $u_{\varepsilon} \leq 0$ in $\bar{B}_{2}$, for $k$ sufficiently large $w_{k} \leq \epsilon M / 2$ in $\bar{B}_{2}$. Define

$$
\bar{w}_{k}=\left\{\begin{array}{l}
\min \left\{w_{k}^{\varepsilon}, v\right\}, \text { in } \bar{B}_{2}, \\
w_{k}^{\varepsilon}, \quad \text { otherwise. }
\end{array}\right.
$$

Then, in view of (4.6), as long as

$$
\varepsilon<\frac{G(0)}{2 M c_{1}}
$$

the function

$$
\bar{w}_{k}(x)=\varepsilon \bar{w}_{k}^{\varepsilon}\left(\frac{x-x_{0}}{\varepsilon}\right)
$$

satisfies

$$
\bar{w}_{k} \in \mathcal{F}, \quad \bar{w}_{k} \leq 0 \quad \text { in } \bar{B}_{\varepsilon}\left(x_{0}\right)
$$

and $\bar{w}_{k} \searrow u$ in $\bar{B}_{\varepsilon}\left(x_{0}\right)$, as desired.

\section{ON THE NON-DEGENERACY OF $u^{+}$}

In this section we prove that $u^{+}$is non-degenerate. As a consequence $F\left(w_{k}\right) \rightarrow$ $F(u)$ locally in Hausdorff distance and $\chi_{\left\{w_{k}>0\right\}} \rightarrow \chi_{\{u>0\}}$ in $L_{l o c}^{1}(\Omega)$.

First, we recall the following standard lemma.

Lemma 5.1. Let $u$ be a Lipschitz function in $\bar{\Omega} \cap B_{1}(0)$ satisfying $\mathcal{L} u=f$, vanishing on $\partial \Omega \cap B_{1}$ and $0 \in \partial \Omega$. Suppose that there exists a positive constant $C$ such that for every $x \in B_{1 / 2} \cap \Omega$

$$
u(x) \geq \operatorname{cdist}(x, \partial \Omega) .
$$

Then there exists a constant $C>0$ such that

$$
\sup _{B_{r}(0)} u \geq C r
$$

for all $r \leq r_{0}$ universal. 
Proof. Let $\operatorname{dist}\left(x_{0}, \partial \Omega\right)=\epsilon$. Then by (5.1) and the Lipschitz continuity of $u$ (say $L=\operatorname{Lip}(u))$

$$
c \epsilon \leq u\left(x_{0}\right) \leq L \epsilon .
$$

We wish to show that there exists $x_{1} \in B_{\varepsilon}\left(x_{0}\right)$ such that

$$
u\left(x_{1}\right) \geq(1+\delta) u\left(x_{0}\right),
$$

with $\delta$ to be specified later.

Assume not, then

$$
v(x):=(1+\delta) u\left(x_{0}\right)-u(x)>0 \quad \text { in } B_{\varepsilon}\left(x_{0}\right)
$$

and solves

$$
\mathcal{L} v=-f \quad \text { in } B_{\varepsilon}\left(x_{0}\right)
$$

By Harnack inequality,

$$
v \leq C(L)\left(\delta u\left(x_{0}\right)+\varepsilon^{2}\|f\|_{\infty}\right) \quad \text { in } \bar{B}_{c(L) \varepsilon}\left(x_{0}\right),
$$

with $c(L)=1-\frac{c}{4 L}$.

Hence, for $\delta<c / 4 L, \varepsilon<c / 4\|f\|_{\infty}$,

$$
v \leq C(L)\left(\delta L \varepsilon+\varepsilon^{2}\|f\|_{\infty}\right) \leq \frac{1}{2} c \varepsilon \leq \frac{u\left(x_{0}\right)}{2} \text { in } \bar{B}_{c(L) \varepsilon}\left(x_{0}\right) .
$$

From the definition of $v$ it follows that

$$
u \geq \frac{c \varepsilon}{2} \quad \text { in } B_{c(L) \varepsilon}\left(x_{0}\right) .
$$

However, from the Lipschitz continuity of $u$ it follows that

$$
u(x) \leq L(1-c(L)) \varepsilon=c \frac{\varepsilon}{4} \quad \text { on } \partial B_{c(L) \varepsilon}\left(x_{0}\right)
$$

a contradiction.

Thus we can construct inductively a sequence of points $x_{k}$ such that

$$
u\left(x_{k+1}\right)=(1+\delta) u\left(x_{k}\right), \quad\left|x_{k+1}-x_{k}\right| \leq C d\left(x_{k}, \partial \Omega\right) .
$$

Then using the fact that $\operatorname{dist}\left(x_{k}, \partial \Omega\right) \sim u\left(x_{k}\right)$ and that $u\left(x_{k}\right)$ grows geometrically we find

$$
\begin{aligned}
\left|x_{k+1}-x_{0}\right| & \leq \sum_{i=0}^{k}\left|x_{i+1}-x_{i}\right| \leq C \sum_{i=0}^{k} \operatorname{dist}\left(x_{i}, \partial \Omega\right) \\
& \leq C \sum_{i=0}^{k} u\left(x_{i}\right) \leq C u\left(x_{k+1}\right) \sim \operatorname{dist}\left(x_{k+1}, \partial \Omega\right) .
\end{aligned}
$$

Hence for a sequence of $r_{k}$ 's of size $u\left(x_{k}\right)$ we have that

$$
\sup _{B_{r_{k}}\left(x_{0}\right)} u \geq c r_{k}
$$

from which we obtain that

$$
\sup _{B_{r}\left(x_{0}\right)} u \geq c r, \quad \text { for all } r \geq\left|x_{0}\right| .
$$

The conclusion follows by letting $x_{0}$ go to 0 . 
Lemma 5.2. There exist universal constants $\bar{r}, \bar{C}>0$, such that

$$
u\left(x_{0}\right) \geq \bar{C} \operatorname{dist}\left(x_{0}, F(u)\right), \quad \text { in }\left\{x \in \Omega^{+}(u): \operatorname{dist}(x, F(u)) \leq \bar{r}\right\} .
$$

Proof. Let $x_{0} \in \Omega^{+}(u), r=\operatorname{dist}\left(x_{0}, F(u)\right)$ with $r \leq \bar{r}$ universal to be specified later. Assume first that

$$
\operatorname{dist}\left(x_{0}, \Omega^{+}(\underline{u})\right)>\frac{r}{2} .
$$

Thus,

$$
\underline{u} \leq 0 \text { in } B_{r / 2}\left(x_{0}\right) .
$$

Let $w_{k} \in \mathcal{F}$ converge uniformly to $u$, say in $B_{R}\left(x_{0}\right), r \leq R$. Let us rescale by $r$ around $x_{0}$ and use the notation (2.1). Then $u_{r}$ solves the free boundary problem

$$
\left\{\begin{array}{l}
\mathcal{L}_{r} u_{r}=r f_{1}^{r} \quad \text { in } \Omega_{r}^{+}\left(u_{r}\right) \\
\mathcal{L}_{r} u_{r}=r f_{2}^{r} \chi_{\left\{u_{r}<0\right\}} \quad \text { in } \Omega_{r}^{-}\left(u_{r}\right) \\
\left(u_{r}\right)_{\nu}^{+}=G_{r}\left(\left(u_{r}\right)_{\nu}^{-}, x, \nu\right) \quad \text { on } F\left(u_{r}\right) .
\end{array}\right.
$$

Moreover $w_{k}^{r}$ converges to $u_{r}$ uniformly in $B_{R / r}$. Clearly, $u_{r}$ is the infimum of all admissible supersolutions to (5.3) (in the sense of Definition 1.2) which are above the rescaling $\underline{u}_{r}(x)=\frac{\underline{u}\left(x_{0}+r x\right)}{r}$.

We wish to prove that

$$
u_{r}(0) \geq \bar{C},
$$

with $\bar{C}>0$ universal, to be specified later. Assume by contradiction

$$
u_{r}(0)<\bar{C} \text {. }
$$

By Harnack inequality in $B_{1} \subset \Omega_{r}^{+}\left(u_{r}\right)$, we have that

$$
u_{r} \leq C(\bar{C}+r M), \quad \text { in } B_{1 / 2}
$$

where $\left\|f_{1}\right\|_{L^{\infty}}=M$. Hence, for $k$ large enough

$$
0<w_{k}^{r} \leq C(\bar{C}+r M) \quad \text { in } B_{1 / 2} .
$$

Now, as in Corollary 4.5 , let $\eta, \xi$ solve the following problems:

$$
\begin{gathered}
\mathcal{L}_{r} \eta=0 \quad \text { in } B_{1 / 2} \backslash \overline{B_{1 / 4}} \\
\eta=0 \quad \text { on } \partial B_{1 / 4}, \quad \eta=1 \quad \text { on } \partial B_{1 / 2}, \\
\mathcal{L}_{r} \xi=-1 \quad \text { in } B_{1 / 2} \backslash \overline{B_{1 / 4}} \\
\xi=0 \quad \text { on } \partial B_{1 / 4}, \quad \xi=0 \quad \text { on } \partial B_{1 / 2} .
\end{gathered}
$$

Call

$$
c_{0}:=\left.\eta_{\nu}\right|_{\partial B_{1 / 4}}>0, \quad c_{1}:=\left.\xi_{\nu}\right|_{\partial B_{1 / 4}}>0
$$

with $\nu$ the unit normal to $\partial B_{1 / 4}$ pointing inward and let $c_{2}>0$ be such that

$$
c_{0} c_{2}<\frac{G(0)}{2} \text {. }
$$

Define,

$$
v:=r M \xi+c_{2} \eta \quad \text { in } B_{1 / 2} \backslash \overline{B_{1 / 4}} .
$$

Then,

$$
\mathcal{L}_{r} v=-r M \quad \text { in } B_{1 / 2} \backslash \overline{B_{1 / 4}} .
$$


Moreover, (say $M>0$ ) if

$$
r<\frac{c_{2}}{2 C M}=r_{1}
$$

and $\bar{C}$ is chosen so that

$$
\bar{C} \leq \frac{c_{2}}{2 C}
$$

then,

$$
0<w_{k}^{r} \leq \frac{c_{2}}{2} \leq v \quad \text { on } \partial B_{1 / 2} .
$$

Now, define

$$
\bar{w}_{r}=\left\{\begin{array}{l}
w_{k}^{r} \quad \text { in } \Omega_{r} \backslash B_{1 / 2} \\
\min \left\{w_{k}^{r}, v\right\} \quad \text { in } B_{1 / 2} \backslash \partial B_{1 / 4} \\
0 \quad \text { in } B_{1 / 4} .
\end{array}\right.
$$

This function is continuous in view of (5.8). Also, from (5.5) and the fact that $w_{k}^{r}>0$ in $B_{1 / 2}$ it follows that

$$
\left\{\begin{array}{l}
\mathcal{L}_{r} \bar{w}_{r} \leq r f_{1}^{r} \quad \text { in } \Omega_{r}^{+}\left(\bar{w}_{r}\right), \\
\mathcal{L}_{r} \bar{w}_{r} \leq r f_{2}^{r} \chi_{\left\{\bar{w}_{r}<0\right\}} \quad \text { in } \Omega_{r}^{-}\left(\bar{w}_{r}\right),
\end{array}\right.
$$

and from $(5.2)$

$$
\bar{w}_{r} \geq \underline{u}_{r} \quad \text { in } \Omega_{r} .
$$

Thus, to assure that $\bar{w}_{r}$ is an admissible supersolution, we need to require that

$$
r M c_{1}+c_{2} c_{0}<G(0) .
$$

In view of (5.4), it is enough to choose

$$
r \leq \frac{G(0)}{2 M c_{1}}=r_{2} .
$$

Thus, for $r \leq \bar{r}:=\min \left\{r_{1}, r_{2}\right\}$ we have reached a contradiction to the minimality of $u_{r}$ since

$$
\bar{w}_{r}(0)=0<u_{r}(0) .
$$

As a consequence of the two lemmas above, we obtain the following corollary.

Lemma 5.3. Let $x \in F(u)$ and let $A$ be a connected component of $\Omega^{+}(u) \cap\left(B_{r}(x) \backslash\right.$ $\left.\bar{B}_{r / 2}(x)\right)$ such that

$$
\bar{A} \cap \partial B_{r / 2}(x) \neq \emptyset, \quad \bar{A} \cap \partial B_{r}(x) \neq \emptyset,
$$

for $r \leq r_{0}$ universal. Then

$$
\sup _{A} u \geq C r .
$$

Moreover

$$
\frac{\left|A \cap B_{r}(x)\right|}{\left|B_{r}(x)\right|} \geq C>0,
$$

where all the constants $C$ depend on $d(x, \partial \Omega)$ and on $\underline{u}$. 


\section{The FUnCtion $u$ IS A SUPERSOLUtion}

In this section we prove that $u$ satisfies part b-(i) in Definition 1.1. First we need to the following preliminary result.

Lemma 6.1. Let $v_{k} \geq 0$ satisfy

$$
\mathcal{L} v_{k} \in L^{\infty} \quad \text { in } B_{2} \cap\left\{v_{k}>0\right\} .
$$

Assume that $v_{k} \rightarrow v$ uniformly in $B_{2}$. Then

$$
\int_{B_{1}} \frac{\left|\nabla v_{k}\right|^{2}}{|x|^{n-2}} d x \rightarrow \int_{B_{1}} \frac{|\nabla v|^{2}}{|x|^{n-2}} d x
$$

Proof. We sketch the proof. Let $V$ be the fundamental solution of the operator $\mathcal{L}$. Then $V \sim|x|^{2-n}($ see $[\mathrm{LSW}])$.

Take a cut-off $\eta \in C_{0}^{\infty}\left(B_{2}\right), \eta=1$ in $B_{1}$. For $w=v$ or $w=v_{k}$ we have:

$$
A(x) \nabla v \cdot \nabla w=\frac{1}{2} \mathcal{L}\left(w^{2}\right)-w \mathcal{L} w .
$$

On the other hand,

$$
\begin{aligned}
\int_{B_{2}} \eta^{2} V \mathcal{L}\left(w^{2}\right) d x & =-\int_{B_{2}} A(x) \nabla\left(\eta^{2} V\right) \cdot \nabla\left(w^{2}\right) d x \\
& =-2 \int_{B_{2}} w \nabla w \cdot\left(A(x)\left[2 \eta V \nabla \eta+\eta^{2} \nabla V\right]\right) d x \\
& =-4 \int_{B_{2} \backslash B_{1}} w \eta V \nabla w \cdot A(x) \nabla \eta d x-\int_{B_{2}} A(x) \nabla V \cdot \nabla\left(w^{2} \eta^{2}\right) d x \\
& +\int_{B_{2} \backslash B_{1}} w^{2} A(x) \nabla V \cdot \nabla\left(\eta^{2}\right) d x \\
& =-4 \int_{B_{2} \backslash B_{1}} w \eta V \nabla w \cdot A(x) \nabla \eta d x-w^{2}(0) \\
& +\int_{B_{2} \backslash B_{1}} w^{2} A(x) \nabla V \cdot \nabla\left(\eta^{2}\right) d x .
\end{aligned}
$$

Thus we deduce from (6.1) that

$$
\int_{B_{2}} \eta^{2} V A(x) \nabla\left(v_{k}-v\right) \cdot \nabla\left(v_{k}-v\right) d x \rightarrow 0 \quad \text { as } k \rightarrow \infty .
$$

From this the desired result follows using ellipticity and the estimate on $V$.

We also need the following variant of the monotonicity formula in Proposition 3.1 (see again $[\mathrm{MP}]$ ). We use the same notation as in Proposition 3.1.

Proposition 6.2. Assume that

$$
u_{i}(x) \leq \sigma(|x|), \quad x \in B_{1}, \quad i=1,2
$$

for a Dini modulus of continuity $\sigma(r)$. Then

$$
\Phi(\rho) \leq[1+\omega(r)] \phi(r)+C \omega(r), \quad 0<\rho \leq r \leq r_{0},
$$

with

$$
\omega(r) \rightarrow 0 \quad \text { as } r \rightarrow 0^{+}
$$

and $C$ depending on $\left\|u_{i}\right\|_{L^{2}\left(B_{1}\right)}, \sigma,[A]_{0, \gamma}$. 
In view of the expansion Lemmas $2.2,2.3$, we only need to prove the next result.

Lemma 6.3. Let $x_{0} \in F(u)$ and

$$
u^{+}(x)=\alpha\left\langle x-x_{0}, \nu\right\rangle^{+}+o\left(\left|x-x_{0}\right|\right),
$$

and

$$
u^{-}=\beta\left\langle x-x_{0}, \nu\right\rangle^{-}+o\left(\left|x-x_{0}\right|\right)
$$

Then,

$$
\alpha \leq G\left(\beta, x_{0}, \nu\right) .
$$

Proof. Let $\left\{w_{k}\right\}_{k \in \mathbb{N}} \subset \mathcal{F}$ be a uniformly decreasing to $u$. As a consequence $w_{k}$ cannot remain strictly positive in a neighborhood of $x_{0}$, say in a ball $B_{r}\left(x_{0}\right)$, for all $k$ large. Otherwise $u$ would be a non-negative solution of $\mathcal{L} u=f_{1}$ in such neighborhood. Then, by standard regularity theory $u \in C^{1, \gamma}$ and $\nabla u\left(x_{0}\right)=0$. Hence, $u_{\nu}^{+}\left(x_{0}\right)=0$ contradicting the non-degeneracy of $u^{+}$.

For each $w_{k}$, let

$$
B_{m, k}=B_{\lambda_{m, k}}\left(x_{0}+\frac{1}{m} \nu\right)
$$

be the largest ball centered at $x_{0}+\frac{1}{m} \nu$ contained in $\Omega^{+}\left(w_{k}\right)$, touching $F\left(w_{k}\right)$ at $x_{m, k}$ where $\nu_{m, k}$ is the unit inward normal of $F\left(w_{k}\right)$ at $x_{m, k}$. Then up to proper subsequences we deduce that

$$
\lambda_{m, k} \rightarrow \lambda_{m}, \quad x_{m, k} \rightarrow x_{m}, \quad \nu_{m, k} \rightarrow \nu_{m}
$$

and $B_{\lambda_{m}}\left(x_{0}+\frac{1}{m} \nu\right)$ touching $F(u)$ at $x_{m}$, with unit inward normal $\nu_{m}$. From the behavior of $u^{+}$, we get that

$$
\begin{gathered}
\left|x_{m}-x_{0}\right|=o\left(\frac{1}{m}\right), \\
\frac{1}{m}+o\left(\frac{1}{m}\right) \leq \lambda_{m} \leq \frac{1}{m}
\end{gathered}
$$

and

$$
\left|\nu_{m}-\nu\right|=o(1)
$$

Now since $w_{k} \in \mathcal{F}$, near $x_{m, k}$ in $B_{m, k}$ :

$$
w_{k}^{+} \leq \alpha_{m, k}\left\langle x-x_{m, k}, \nu_{m, k}\right\rangle^{+}+o\left(\left|x-x_{m, k}\right|\right)
$$

and in $\Omega \backslash B_{m, k}$

$$
w_{k}^{-} \geq \beta_{m, k}\left\langle x-x_{m, k}, \nu_{m, k}\right\rangle^{-}+o\left(\left|x-x_{m, k}\right|\right)
$$

with

$$
0 \leq \alpha_{m, k} \leq G\left(\beta_{m, k}, x_{m, k}, \nu_{m, k}\right),
$$

(by Lemma 2.1 the touching occurs at a regular point, for $m, k$ large.) We know that

$$
w_{k}^{+} \geq u^{+} \geq \alpha\left\langle x-x_{0}, \nu\right\rangle^{+}+o\left(\left|x-x_{0}\right|\right),
$$

hence

$$
\underline{\alpha}_{m}=\liminf _{k \rightarrow \infty} \alpha_{m, k} \geq \alpha-\epsilon_{m}
$$

and $\epsilon_{m} \rightarrow 0$, as $m \rightarrow \infty$. We have to prove that

$$
\underline{\beta}=\liminf _{m, k \rightarrow+\infty} \beta_{m, k} \leq \beta .
$$


To do this we argue as follows. If $\underline{\beta}=0$ there is nothing to prove. Hence let $\beta_{m, k}>0$. Given $r, \bar{x}, v$ denote by

$$
\Phi_{r}(\bar{x}, v)=r^{-4} \int_{B_{r}(\bar{x})} \frac{\left|\nabla v^{+}\right|^{2}}{|x-\bar{x}|^{n-2}} d x \int_{B_{r}(\bar{x})} \frac{\left|\nabla v^{-}\right|^{2}}{|x-\bar{x}|^{n-2}} d x .
$$

From (3.2) in Theorem 3.5 we obtain that ( $\rho$ small)

$$
\Phi_{\rho}\left(x_{m, k}, w_{k}\right) \geq c_{n} \alpha_{m, k}^{2} \beta_{m, k}^{2}+o(1)
$$

with $o(1) \rightarrow 0$ as $\rho \rightarrow 0$.

Thus, using Proposition 6.2 and letting $\rho \rightarrow 0$, we get that ( $r$ small)

$$
(1+\omega(r)) \Phi_{r}\left(x_{m, k}, w_{k}\right)+C \omega(r) \geq c_{n} \alpha_{m, k}^{2} \beta_{m, k}^{2} .
$$

We remark that the $w_{k}^{ \pm}$satisfy the assumptions of Proposition 6.2. Indeed the $w_{k}^{+}$are equilipschitz. To obtain a uniform modulus of continuity for the $w_{k}^{-}$notice that, the $F\left(w_{k}^{-}\right)$have an exterior tangent ball at $x_{m, k}$ of size $1 / m$. Thus in a neighborhood of $x_{m, k}$ of size $2 / m$ a modulus of continuity independent of $k$ can be obtained building an appropriate barrier. Outside such a neighborhood, the $w_{k}^{-}$ inherit the modulus of continuity of the $u^{-}$, because $w_{k}^{-}$converges to $u^{-}$uniformly.

Now from (3.2), we also have that

$$
\Phi_{r}\left(x_{0}, u\right) \geq c_{n} \alpha^{2} \beta^{2}+o(1) \quad \text { as } r \rightarrow 0^{+} .
$$

On the other hand, since $u^{ \pm}$are Lipschitz continuous, for $\delta$ small and $r$ small depending on $\delta$

$$
\int_{B_{r}\left(x_{0}\right)}\left|\nabla u^{+}\right|^{2} d x=\int_{B_{1}}\left|\nabla u_{r}^{+}\right|^{2} d x \leq \alpha^{2}\left|B_{1} \cap\{x \cdot \nu>\delta\}\right|+O(\delta)+o(1)
$$

Analogously,

$$
\int_{B_{r}\left(x_{0}\right)}\left|\nabla u^{-}\right|^{2} d x \leq \beta^{2}\left|B_{1} \cap\{x \cdot \nu>\delta\}\right|+O(\delta)+o(1) .
$$

By Remark 3.2,

$$
\Phi_{r}\left(x_{0}, u\right)=\Phi_{1}\left(0, u_{r}\right) \leq \frac{1}{4} \alpha^{2} \beta^{2}\left|B_{1} \cap\{x \cdot \nu>\delta\}\right|^{2}+O(\delta)+o(1) .
$$

This, together with (6.3) gives that

$$
\lim _{r \rightarrow 0^{+}} \Phi_{r}\left(x_{0}, u\right)=c_{n} \alpha^{2} \beta^{2} .
$$

Moreover, since $x_{m, k} \rightarrow x_{m}$ and $w_{k} \rightarrow u$ uniformly, we get from Lemma 6.1 that

$$
\lim _{k \rightarrow \infty} \Phi_{r}\left(x_{m, k}, w_{k}\right)=\Phi_{r}\left(x_{m}, u\right) .
$$

In particular, it follows from that for every $\epsilon>0$ there exist $r>0$ small, and $m, k$ large (all depending on $\varepsilon$ ) such that

$$
\Phi_{r}\left(x_{m, k}, w_{k}\right) \leq c_{n} \alpha^{2} \beta^{2}+\epsilon .
$$

Applying (6.2) and recalling that

$$
\liminf _{m, k \rightarrow \infty} \alpha_{m, k} \geq \alpha,
$$

it follows that $\underline{\beta} \leq \beta$, because $\alpha>0$ (by non-degeneracy.) 


\section{The FUnCtion $u$ is A SUbSOlUtion}

In this section we want to show that $u$ satisfies part b.(ii) in Definition 1.1, that is if $x_{0} \in F(u)$ is a regular point from the left with touching ball $B \subset \Omega^{-}(u)$, then near to $x_{0}$

$$
u^{-}(x)=\beta\left\langle x-x_{0}, \nu\right\rangle^{-}+o\left(\left|x-x_{0}\right|\right), \quad \beta \geq 0
$$

in $B$, and

$$
u^{+}(x)=\alpha\left\langle x-x_{0}, \nu\right\rangle^{+}+o\left(\left|x-x_{0}\right|\right), \quad \alpha \geq 0
$$

in $\Omega \backslash B$ with $\alpha \geq G\left(\beta, x_{0}, \nu\right)$.

Notice that, even if $\beta=0$, then $\Omega^{+}(u)$ and $\Omega^{-}(u)$ are tangent to $\left\{\left\langle x-x_{0}, \nu\right\rangle=0\right\}$ at $x_{0}$ since $u^{+}$is non-degenerate. Thus $u$ has a full asymptotic development as in the next lemma.

Lemma 7.1. Assume that near $x_{0} \in F(u)$,

$$
u(x)=\alpha\left\langle x-x_{0}, \nu\right\rangle^{+}-\beta\left\langle x-x_{0}, \nu\right\rangle^{-}+o\left(\left|x-x_{0}\right|\right),
$$

with $\alpha>0, \beta \geq 0$. Then

$$
\alpha \geq G\left(\beta, x_{0}, \nu\right)
$$

Proof. Assume by contradiction that $\alpha<G\left(\beta, x_{0}, \nu\right)$. We will show that in this case we can build a supersolution $w \in \mathcal{F}$ which is strictly smaller than $u$ at some point, contradicting the minimality of $u$. Let $u_{0}$ be the two-plane solution, i.e.

$$
u_{0}(x):=\lim _{r \rightarrow 0} \frac{u\left(x_{0}+r x\right)}{r}=\alpha\langle x, \nu\rangle^{+}-\beta\langle x, \nu\rangle^{-} .
$$

Suppose that $\alpha \leq G\left(\beta, x_{0}, \nu\right)-\delta_{0}$ with $\delta_{0}>0$. Fix $\zeta=\zeta\left(\delta_{0}\right)$, to be made precise later.

In view of Corollary 4.5, we can find $w_{k} \searrow u$ uniformly and for $r$ small, $k$ large the rescaling $w_{k, r}$ satisfies the following:

(i) if $\beta>0$, then $w_{k, r}(x) \leq u_{0}+\zeta \min \{\alpha, \beta\}$ on $\partial B_{1}$,

(ii) if $\beta=0$, then $w_{k, r}(x) \leq u_{0}+\alpha \zeta$ on $\partial B_{1}$, and

$$
w_{k, r}(x) \leq 0, \quad \text { in }\{\langle x, \nu\rangle<-\zeta\} \cap \bar{B}_{1} .
$$

In particular,

$$
w_{k, r}(x) \leq u_{0}(x+\zeta \nu) \quad \text { on } \partial B_{1} .
$$

If $\beta>0$, let $v$ satisfy (using the notation in (2.1))

$$
\left\{\begin{array}{l}
\mathcal{L}_{r} v=r f_{1}^{r}, \quad\{\langle x, \nu\rangle>-\zeta+\epsilon \phi(x)\} \\
\mathcal{L}_{r} v=r f_{2}^{r}, \quad\{\langle x, \nu\rangle<-\zeta+\epsilon \phi(x)\} \\
v(x)=0, \quad\{\langle x, \nu\rangle=-\zeta+\epsilon \phi(x)\} \\
v(x)=u_{0}(x+\zeta \nu), \quad \partial B_{1}
\end{array}\right.
$$

where $\phi \geq 0$ is a cut-off function, $\phi \equiv 0$ outside $B_{1 / 2}, \phi \equiv 1$ inside $B_{1 / 4}$.

For $\beta=0$, replace the second equation with $v=0$.

Along the new free boundary, $F(v)=\{\langle x, \nu\rangle=-\zeta+\epsilon \phi(x)\}$ we have the following estimates:

$$
\left|v_{\nu}^{+}-\alpha\right| \leq c(\varepsilon+\zeta)+C r, \quad\left|v_{\nu}^{-}-\beta\right| \leq c(\varepsilon+\zeta)+C r
$$

with $c, C$ universal.

Indeed,

$$
v^{+}-\alpha\langle x, \nu\rangle^{+}
$$


is solution of

$$
\mathcal{L}_{r}\left(v-\alpha\langle x, \nu\rangle^{+}\right)=g_{r} \quad g_{r}:=r\left(f_{1}^{r}-\alpha \operatorname{div}\left(A_{r} \nu\right)\right) .
$$

Thus, by standard $C^{1, \gamma}$ estimates

$$
\left|v_{\nu}^{+}-\alpha\right| \leq C\left(\left\|v-\alpha\langle x, \nu\rangle^{+}\right\|_{\infty}+[-\gamma+\epsilon \phi]_{1, \gamma}+r\left\|f_{1}\right\|_{\infty}+r[A]_{0, \gamma}\right),
$$

which gives the desired bound. Similarly, one gets the bound for $v_{\nu}^{-}$.

Hence, since $\alpha \leq G\left(\beta, x_{0}, \nu\left(x_{0}\right)\right)-\delta_{0}$, say for $\varepsilon=2 \zeta$ and $\zeta, r$ small depending on $\delta_{0}$

$$
v_{\nu}^{+}<G\left(v_{\nu}^{-}, x_{0}, \nu\right)
$$

and the function,

$$
\bar{w}_{k}=\left\{\begin{array}{l}
\min \left\{w_{k}, \lambda v\left(\frac{x-x_{0}}{\lambda}\right)\right\} \\
w_{k} \text { in } \Omega \backslash B_{\lambda}^{\lambda}\left(x_{0}\right),
\end{array}\right.
$$

is still in $\mathcal{F}$. However, the set

$$
\{\langle x, \nu\rangle \leq-\zeta+\epsilon \phi\}
$$

contains a neighborhood of the origin, hence rescaling back $x_{0} \in \Omega^{-}\left(\bar{w}_{k}\right)$. We get a contradiction since $x_{0} \in F(u)$ and $\Omega^{+}(u) \subseteq \Omega^{+}\left(\bar{w}_{k}\right)$.

\section{The SIZE OF THE REDUCED BOUNDARY}

In this section we prove our regularity Theorem 1.4. First, we need the following standard result.

Theorem 8.1. Let $u$ be a solution to (1.1), such that $u$ is Lipschitz and nondegenerate. Let $x_{0} \in F(u) \cap B_{1}$ and $0<\epsilon<\delta<1$. Then the following quantities are comparable with $\delta^{n-1}$ :

(i) $\frac{1}{\epsilon}\left|\{0<u<\epsilon\} \cap B_{\delta}\left(x_{0}\right)\right|$,

(ii) $\frac{1}{\epsilon}\left|\mathcal{N}_{\epsilon}(F(u)) \cap B_{\delta}\left(x_{0}\right)\right|$,

(iii) $N \epsilon^{n-1}$, where $N$ is the number of any family of balls of radius $\epsilon$ with finite overlapping covering $F(u) \cap B_{\delta}\left(x_{0}\right)$.

Proof. We follow the proof of Lemma 10 in [C3]. It suffices to show that

$$
\begin{gathered}
\int_{\{0<u<\varepsilon\} \cap B_{\delta}\left(x_{0}\right)}|\nabla u|^{2} \sim \varepsilon \delta^{n-1}, \\
\int_{B_{\varepsilon}\left(x_{0}\right)}|\nabla u|^{2} \sim \varepsilon^{n} .
\end{gathered}
$$

Then, the argument is the same as in the above cited lemma. We notice that the constants in these comparisons depend on the Lipschitz and non-degenerate bounds for $u$, say $C_{1}, c_{1}$. In what follows, dependance on $C_{1}, c_{1}$ (as well as the other universal parameters of the problem) is understood and constants depending on these parameters are still called universal.

Inequality (8.2) follows from standard methods (using Poincare's inequality for the lower bound). Indeed, since $u^{+}$is Lipschitz and non-degenerate

$$
\sup _{B_{\varepsilon}\left(x_{0}\right)} u^{+} \sim \varepsilon, \quad \inf _{B_{\varepsilon}\left(x_{0}\right)} u^{+}=0 .
$$


To prove (8.1), we rescale

$$
u_{\delta}(x)=\frac{u\left(x_{0}+\delta x\right)}{\delta}, \quad x \in B_{1},
$$

and use the notation in (2.1). Let $u_{\varepsilon, s}=\max \left(s / \delta, \min \left(u_{\delta}, \varepsilon / \delta\right)\right), 0<s<\varepsilon$. Then,

$$
\begin{aligned}
& -\delta \int_{B_{1}} f_{1}^{\delta} u_{\epsilon, s}=-\int_{B_{1}} u_{\epsilon, s} \mathcal{L}_{\delta} u_{\delta}^{+} \\
& =\int_{B_{1}}\left\langle A_{\delta}(x) \nabla u_{\delta}^{+}, \nabla u_{\epsilon, s}^{+}\right\rangle d x-\int_{\partial B_{1}}\left\langle A(x) \nabla u_{\delta}^{+}, \nu\right\rangle u_{\epsilon, s} d \mathcal{H}^{n-1} \\
& =\int_{B_{1} \cap\left\{0<s / \delta<u_{\delta}<\epsilon / \delta\right\}}\left\langle A_{\delta}(x) \nabla u_{\delta}, \nabla u_{\delta}\right\rangle d x-\int_{\partial B_{1}}\left\langle A_{\delta}(x) \nabla u_{\delta}^{+}, \nu\right\rangle u_{\epsilon, s} d \mathcal{H}^{n-1},
\end{aligned}
$$

because $\nabla u_{\epsilon, s}=\nabla u_{\delta} \cdot \chi_{\left\{s / \delta<u_{\delta}<\epsilon / \delta\right\}}$.

Hence by ellipticity, using that $u^{+}$is Lipschitz and $f_{1}$ is bounded we get $(\delta<1)$

$$
\int_{B_{1} \cap\left\{0<s / \delta<u_{\delta}<\epsilon / \delta\right\}}\left|\nabla u_{\delta}\right|^{2} d x \leq C \frac{\epsilon}{\delta},
$$

with $C$ universal. Letting $s \rightarrow 0$ and rescaling back, we obtain the upper bound in (8.1).

To obtain the lower bound, let $V$ be the solution to

$$
\left\{\begin{array}{l}
\mathcal{L}_{\delta} V=-\frac{\chi_{B_{\sigma}}}{\left|B_{\sigma}\right|}, \quad \text { in } B_{1} \\
V=0, \quad \text { on } \partial B_{1}
\end{array}\right.
$$

with $\sigma$ to be chosen later. By standard estimates, see for example [GT], $V \leq C(\sigma)$ and $-\left\langle A_{\delta} \nabla V, \nu\right\rangle \sim C^{*}$ on $\partial B_{1}$. By Green formula $\left(u_{\varepsilon}=u_{\varepsilon, 0}\right)$

$$
\int_{B_{1}}\left(\mathcal{L}_{\delta} V\right) \frac{u_{\delta}^{+} u_{\epsilon}}{\epsilon}-\left(\mathcal{L}_{\delta} \frac{u_{\delta}^{+} u_{\epsilon}}{\epsilon}\right) V=\int_{\partial B_{1}} \frac{u_{\delta}^{+} u_{\epsilon}}{\epsilon}\left\langle A_{\delta} \nabla V, \nu\right\rangle d \mathcal{H}^{n-1}
$$

because $V=0$ on $\partial B_{1}$. We estimate

$$
\delta\left|\int_{B_{1}}\left(\mathcal{L}_{\delta} V\right) \frac{u_{\delta}^{+} u_{\epsilon}}{\epsilon} d x\right|=\left|f_{B_{\sigma}} \frac{u_{\delta}^{+} u_{\epsilon}}{\epsilon} d x\right| \leq \bar{C} \sigma,
$$

because $u$ is Lipschitz, $0 \leq u_{\epsilon} \leq \epsilon / \delta$. From (8.4) and (8.5) and the fact that $\left\langle A_{\delta} \nabla V, \nu\right\rangle \sim-C^{*}$ on $\partial B_{1}$ we deduce that

$$
\begin{aligned}
\delta \int_{B_{1}}\left(\mathcal{L}_{\delta} \frac{u_{\delta}^{+} u_{\epsilon}}{\epsilon}\right) V d x & \geq-\bar{C} \sigma-\delta \int_{\partial B_{1}} \frac{u_{\delta}^{+} u_{\epsilon}}{\epsilon}\left\langle A_{\delta} \nabla V, \nu\right\rangle d \mathcal{H}^{n-1} \\
& \geq-\bar{C} \sigma+C^{*} \int_{\partial B_{1}} \frac{u_{\delta}^{+} u_{\epsilon}}{\epsilon} d \mathcal{H}^{n-1} .
\end{aligned}
$$

Thus using that $u^{+}$is non-degenerate and choosing $\sigma$ small enough we get that

$$
\delta \int_{B_{1}}\left(\mathcal{L}_{\delta} \frac{u_{\delta}^{+} u_{\epsilon}}{\epsilon}\right) V d x \geq \tilde{C} .
$$

On the other hand in $\left\{0<u_{\delta}^{+}<\epsilon / \delta\right\}$

$$
\mathcal{L}_{\delta}\left(\frac{u_{\delta}^{+} u_{\epsilon}}{\epsilon}\right)=\frac{2 \delta}{\epsilon} u_{\varepsilon} f_{1}^{\delta}+\frac{1}{\epsilon}\left\langle A_{\delta} \nabla u_{\delta}, \nabla u_{\delta}\right\rangle .
$$


Combining (8.6)-(8.7) and using the ellipticity of $A_{\delta}$ we get that

$$
\frac{2 \delta^{2}}{\epsilon} \int_{B_{1}} u_{\varepsilon} f_{1}^{\delta} V+\frac{\delta \Lambda}{\epsilon} \int_{B_{1}}\left|\nabla u_{\delta}\right|^{2} V \geq \bar{C} .
$$

From the estimate on $V$ we obtain that for $\delta$ small enough

$$
\frac{\delta}{\epsilon} \int_{B_{1}}\left|\nabla u_{\delta}\right|^{2} V \geq C
$$

for some $C$ universal. Rescaling, we obtain the desired lower bound.

Let $u$ be the minimal solution constructed in Theorem 1.3. Then, Theorem 8.1 above implies that $\Omega^{+}(u) \cap B_{r}(x), x \in F(u)$ is a set of finite perimeter. Next we show that in fact this perimeter is equivalent to $r^{n-1}$, and thus conclude the proof of Theorem 1.4. Constant depending possibly on the Lipschitz and non-degeneracy bounds for $u$ are still called universal.

Theorem 8.2. Let $u$ be the minimal solution in Theorem 1.3. Then, the reduced boundary of $\Omega^{+}(u)$ has positive density in $\mathcal{H}^{n-1}$ measure at any point of $F(u)$, i.e. for $r<r_{0}, r_{0}$ universal

$$
\mathcal{H}^{n-1}\left(F^{*}(u) \cap B_{r}(x)\right) \geq c r^{n-1},
$$

for every $x \in F(u)$.

Proof. The proof follows the lines of Corollary 4 in [C3]. Let $w_{k} \in \mathcal{F}, w_{k} \searrow u$ in $\bar{B}_{1}$ and $\mathcal{L} w_{k}=f_{1}$ in $\Omega^{+}(u)$. Let $x_{0} \in F(u)$. As usual, we rescale and use the notation in (2.1):

$$
u_{r}(x)=\frac{u\left(x_{0}+r x\right)}{r}, \quad w_{k, r}=\frac{w_{k}\left(x_{0}+r x\right)}{r} \quad x \in B_{1} .
$$

As in Theorem 8.1, we use the auxiliary function $V$ such that

$$
\left\{\begin{array}{l}
\mathcal{L}_{r} V=-\frac{\chi_{B_{\sigma}}}{\left|B_{\sigma}\right|}, \quad \text { in } B_{1} \\
V=0, \quad \text { on } \partial B_{1}
\end{array}\right.
$$

Since $\nabla w_{k, r}$ is a continuous vector field in $\overline{\Omega_{r}^{+}\left(u_{r}\right) \cap B_{1}}$, we can use it to test for perimeter. Denoting for simplicity $w_{k, r}=w$, we get

$$
\begin{aligned}
& \int_{B_{1} \cap \Omega_{r}^{+}\left(u_{r}\right)}\left(V \mathcal{L}_{r} w-w \mathcal{L}_{r} V\right) \\
& =\int_{F^{*}\left(u_{r}\right) \cap B_{1}}\left(V\left\langle A_{r} \nabla w, \nu\right\rangle-w\left\langle A_{r} \nabla V, \nu\right\rangle\right) d \mathcal{H}^{n-1}-\int_{\partial B_{1} \cap \Omega_{r}^{+}\left(u_{r}\right)} w\left\langle A_{r} \nabla V, \nu\right\rangle d \mathcal{H}^{n-1} .
\end{aligned}
$$

Using estimates for $V$ and the fact that the $w_{k}$ are uniformly Lipschitz, we get that

$$
\left|\int_{F^{*}\left(u_{r}\right) \cap B_{1}} V\left\langle A_{r} \nabla w, \nu\right\rangle d \mathcal{H}^{n-1}\right| \leq C(\sigma) \mathcal{H}^{n-1}\left(F^{*}\left(u_{r}\right) \cap B_{1}\right) .
$$

As in [C3] we have, as $k \rightarrow \infty$

$$
\begin{gathered}
\int_{F^{*}\left(u_{r}\right) \cap B_{1}} w\left\langle A_{r} \nabla V, \nu\right\rangle d \mathcal{H}^{n-1} \rightarrow 0, \\
\int_{\partial B_{1} \cap \Omega_{r}^{+}\left(u_{r}\right)} w\left\langle A_{r} \nabla V, \nu\right\rangle d \mathcal{H}^{n-1} \rightarrow \int_{\partial B_{1}} u_{r}^{+}\left\langle A_{r} \nabla V, \nu\right\rangle d \mathcal{H}^{n-1}
\end{gathered}
$$


and

$$
-\int_{B_{1} \cap \Omega_{r}^{+}\left(u_{r}\right)} w \mathcal{L}_{r} V \rightarrow f_{B_{\sigma}} u_{r}^{+} .
$$

Passing to the limit in (8.9) and using all of the above we get

$$
\begin{aligned}
& \left|r \int_{B_{1} \cap \Omega^{+}\left(u_{r}\right)} V f_{1}^{r}+f_{B_{\sigma}} u_{r}^{+}+\int_{\partial B_{1}} u_{r}^{+}\left\langle A_{r} \nabla V, \nu\right\rangle d \mathcal{H}^{n-1}\right| \\
& \leq C(\sigma) \mathcal{H}^{n-1}\left(F^{*}\left(u_{r}\right) \cap B_{1}\right) .
\end{aligned}
$$

Since $u$ is Lipschitz and non-degenerate, for $\sigma$ small

$$
f_{B_{\sigma}} u_{r}^{+} \leq \bar{C} \sigma
$$

and using the estimate for $\left\langle A_{r} \nabla V, \nu\right\rangle$

$$
-\int_{\partial B_{1}} u_{r}^{+}\left\langle A_{r} \nabla V, \nu\right\rangle d \mathcal{H}^{n-1} \geq \bar{c}>0 .
$$

Also, since $f_{1}^{r}$ is bounded

$$
\int_{B_{1} \cap \Omega_{r}^{+}\left(u_{r}\right)} V f_{1}^{r} \leq \bar{C}(\sigma)
$$

Hence choosing first $\sigma$ and then $r$ sufficiently small we get that the left-hand-side in equation (8.11) is larger than a constant $\tilde{C}$, which concludes our proof.

\section{REFERENCES}

[C3] Caffarelli L.A., A Harnack inequality approach to the regularity of free boundaries. III. Existence theory, compactness, and dependence on X, Ann. Scuola Norm. Sup. Pisa Cl. Sci. (4) 15 (1988), no. 4, 583602 (1989).

[CS] Caffarelli L. A., Salsa S., A Geometric Approach to Free Boundary Problems, Graduate Studies in Mathematics, vol. 682005.

[DFSs1] De Silva D., Ferrari F., Salsa S., On two phase free boundary problems governed by elliptic equations with distributed sources, Discrete Contin. Dyn. Syst. Ser. S 7, no. 4, 673-693 (2014).

[DFS4] De Silva D., Ferrari F., Salsa S., The regularity of flat free boundaries for a nonhomogeneous two-phase problem in divergence form, in preparation.

[GT] Gilbarg D., Trudinger N. S., Elliptic partial differential equations of second order. Classics in Mathematics. Springer-Verlag, Berlin, (2001).

[KS] Kinderlehrer D., Stampacchia G., An introduction to variational inequalities and their applications, Classics in Applied Mathematics, SIAM 2000.

[LSW] Littman W., Stampacchia G.., Weinberger H. F., Regular points for elliptic equations with discontinuous coefficients. Ann. Scuola Norm. Sup. Pisa 17, 43-77, (1963).

[MP] Matevosyan N., Petrosyan A., Almost monotonicity formulas for elliptic and parabolic operators with variable coefficients, Comm. Pure Appl. Math., 64(2):271-311, 2011.

[TE] Teixeira E. V., A variational treatment for general elliptic equations of the flame propagation type: regularity of the free boundary, Ann. Inst. H. Poincare Anal. Non Lineaire 25 (2008), no. 4, 633-658.

[TZ] Teixeira E. V., Zhang L., Monotonicity theorems for Laplace Beltrami operator on Riemannian manifolds. Adv. Math. 226 (2011), no. 2, 1259-1284.

[T] Troianiello G. M., Elliptic Differential Equations and Obstacle Problems, Springer Science \& Business Media, Jul 31, 1987. 
Department of Mathematics, Barnard College, Columbia University, New York, Ny 10027

E-mail address: desilva@math.columbia.edu

Dipartimento di Matematica dell' Università, Piazza di Porta S. Donato, 5, 40126 Bologna, ItALy.

E-mail address: fausto.ferrari@unibo.it

Dipartimento di Matematica del Politecnico, Piazza Leonardo da Vinci, 32, 20133 Milano, Italy.

E-mail address: sandro.salsa@polimi.it 